\title{
Electrochromic Windows: \\ Process and Fabrication Improvements for Lower Total Costs
}

\author{
Final Report
}

Type of Report:

Reporting Period Start Date:

Reporting Period End Date:

Principal Authors:

Date Report was Issued:

DOE Award Number:

Submitting Organization:
Final

September 30, 2003

September 20, 2006

Dr. Mark Burdis

Dr. Neil Sbar

March 31, 2009

DE-FC26-03NT41952

SAGE Electrochromics, Inc

One Sage Way

Faribault, MN 55021

507-331-4848

www.sage-ec.com 


\section{Disclaimer:}

“This report was prepared as an account of work sponsored by an agency of the United States Government. Neither the United States Government nor any agency thereof, nor any of their employees, makes any warranty, express or implied, or assumes any legal liability or responsibility for the accuracy, completeness, or usefulness of any information, apparatus, product, or process disclosed, or represents that its use would not infringe privately owned rights. Reference herein to any specific commercial product, process, or service by trade name, trademark, manufacturer, or otherwise does not necessarily constitute or imply its endorsement, recommendation, or favoring by the United States Government or any agency thereof. The views and opinions of authors expressed herein do not necessarily state or reflect those of the United States Government or any agency thereof.” 


\section{Abstract}

The overall goal with respect to the U.S. Department of Energy (DOE) is to achieve significant national energy savings through maximized penetration of EC windows into existing markets so that the largest cumulative energy reduction can be realized. The speed with which EC windows can be introduced and replace current IGU's (and current glazings) is clearly a strong function of cost. Therefore, the aim of this project was to investigate possible improvements to the SageGlass ${ }^{\circledR}$ EC glazing products to facilitate both process and fabrication improvements resulting in lower overall costs. The project was split into four major areas dealing with improvements to the electrochromic layer, the capping layer, defect elimination and general product improvements.

Significant advancements have been made in each of the four areas. These can be summarized as follows:

- Plasma assisted deposition for the electrochromic layer was pursued, and several improvements made to the technology for producing a plasma beam were made. Functional EC devices were produced using the new technology, but there are still questions to be answered regarding the intrinsic properties of the electrochromic films produced by this method.

- The capping layer work was successfully implemented into the existing SageGlass ${ }^{\circledR}$ product, thereby providing a higher level of transparency and somewhat lower reflectivity than the 'standard' product.

- Defect elimination is an ongoing effort, but this project spurred some major defect reduction programs, which led to significant improvements in yield, with all the implicit benefits afforded. In particular, major advances were made in the development of a new bus bar application process aimed at reducing the numbers of 'shorts' developed in the finished product, as well as making dramatic improvements in the methods used for tempering the glass, which had previously been seen to produce a defect which appeared as a pinhole.

- Improvements have also been made to the overall product to enhance the appearance and market acceptability. These include: (i) increasing the active electrochromic area to enable window manufacturers to install the SageGlass ${ }^{\circledR}$ IGU's into a variety of different framing systems, (ii) implementing a Pb free solder system for the electrical interconnections, (iii) development of a wire routing scheme to allow installation of SageGlass ${ }^{\circledR}$ units into a variety of different framing systems.

This project has advanced the development of electrochromic glazing significantly, thereby advancing the introduction of the product and all the benefits of such a technology. 


\section{Table of Contents}

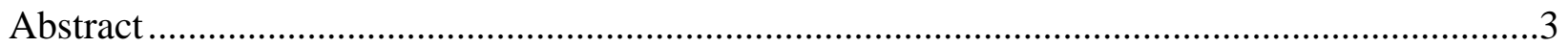

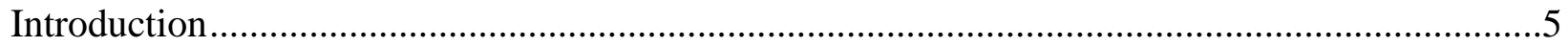

Description and Benefits of Electrochromic Windows .........................................................5

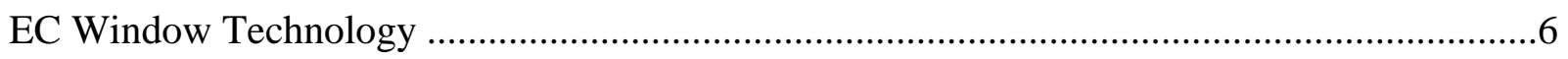

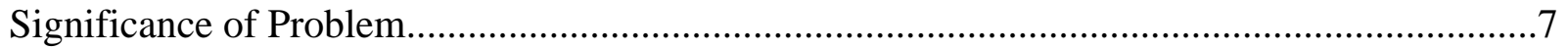

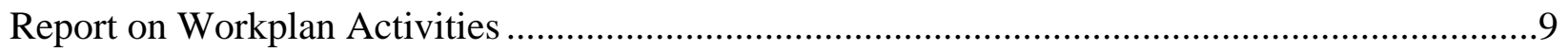

I. Improved EC Layer Deposition Process .........................................................................9

I.1 Deposit of Baseline and Ion Assisted EC Layers .......................................................10

I.2 Characterization of Baseline and Ion Assisted EC films.............................................15

I.3 Fabricate LBNL films into small devices..............................................................18

II. Thin Film Capping Layer.........................................................................................20

II.1 Install Target, Measure Film Properties .................................................................20

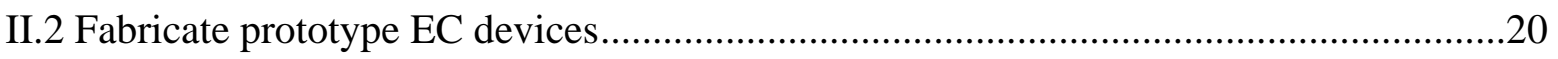

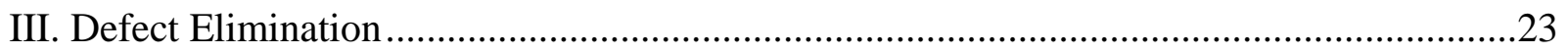

III.1.1 Survey and Optimize Tempering Factors ...........................................................23

III.2.1 Develop Method to Generate Defects ...............................................................27

III.3 Evaluate Ion Exchange Layer ...............................................................................28

III.5 Post EC Layer Chemical Treatment ....................................................................29

III.7 Develop techniques for repairing or excising electrical short defects ..........................32

III.7.1 X-Y Stage and Algorithm Development for Elimination of Shorts ..........................32

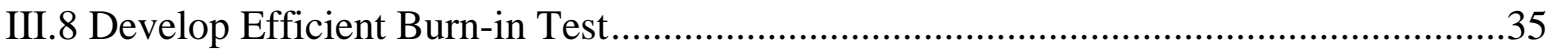

IV. Product Improvement to Increase Acceptance to OEM Customers ................................37

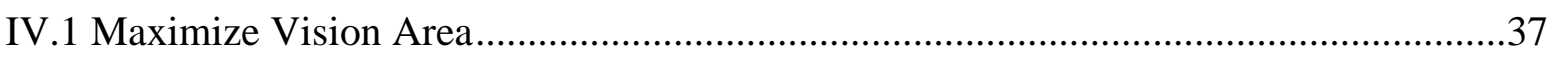

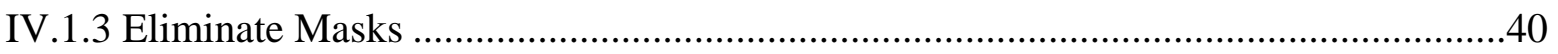

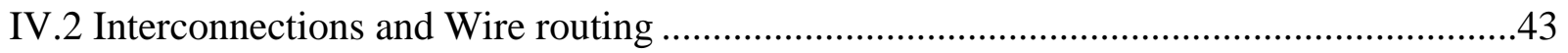

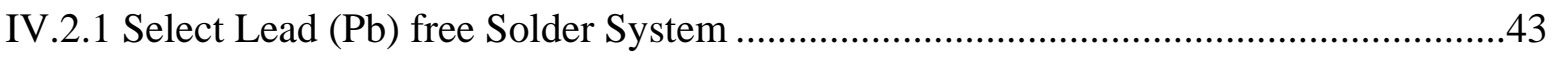

IV.2.4 Develop wire routing scheme .....................................................................44

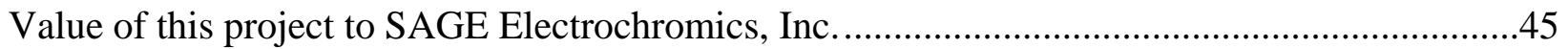

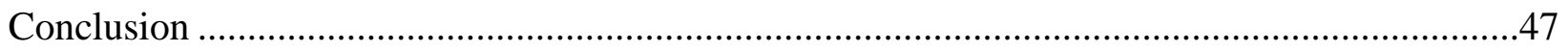




\section{Introduction}

This report is divided into four main sections. The first introductory section deals briefly with background information intended to place the rest of the report in context. It includes a general

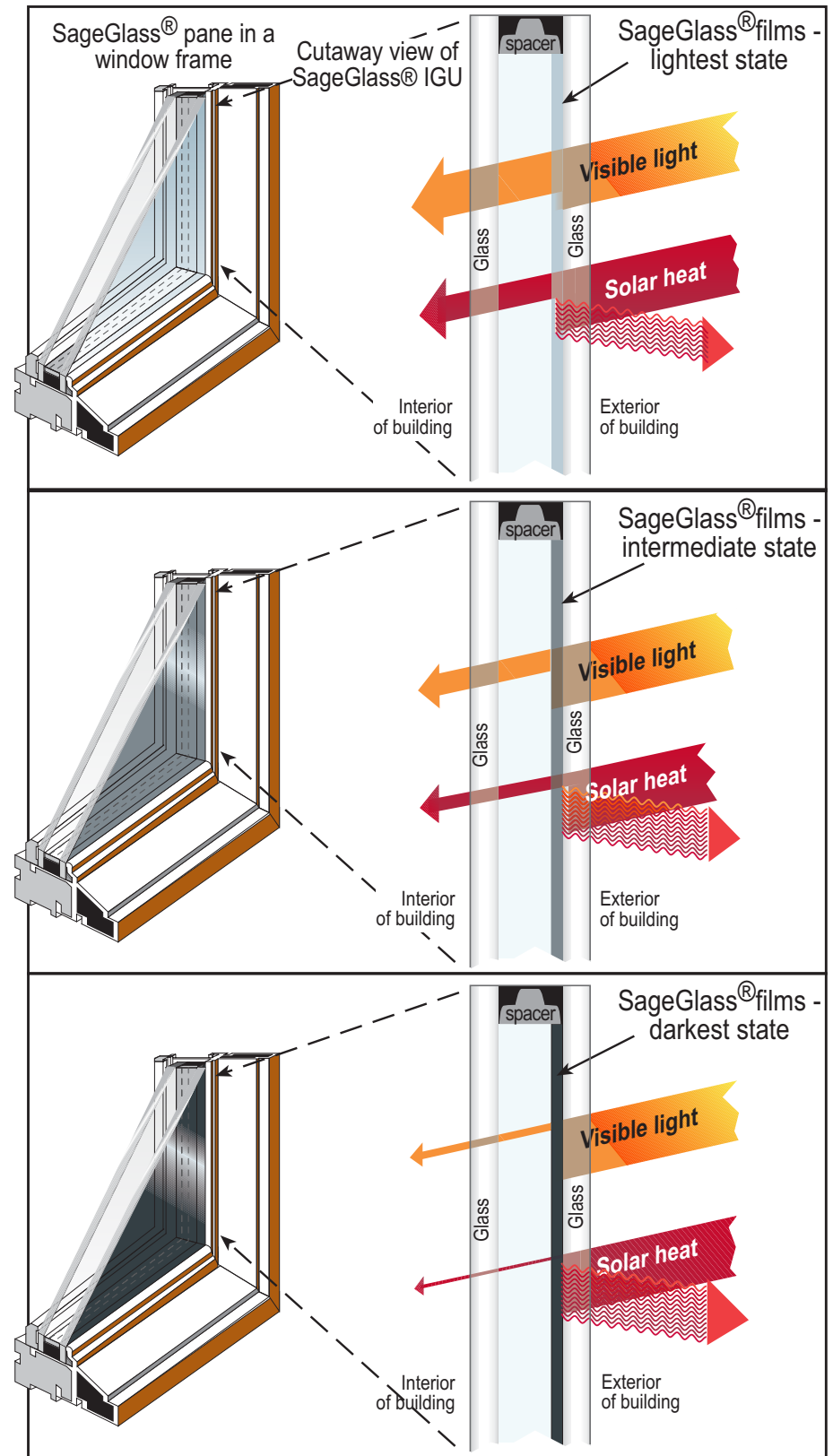

Figure 1 - Schematic of the principle of operation of a SageGlass EC window. introduction to electrochromic technology, followed by information on the tasks included in this project. There then follows a detailed description of work carried out on each of the tasks.

\section{Description and Benefits of Electrochromic Windows}

There is a need to improve the energy efficiency of building envelopes as they are the primary factor governing the heating, cooling, lighting and ventilation requirements of buildings - influencing 53\% of building energy usage. In particular, windows contribute significantly to the overall energy performance of building envelopes, thus there is a need to develop advanced energy efficient window and glazing systems.

The electrochromic (EC) window represents the next generation of advanced glazing technology that will both reduce the energy consumed in buildings, and improve the overall comfort of the occupants. "Switchable" EC windows provide, on demand, dynamic control of

visible light, solar heat gain, and glare without blocking the view. As exterior light levels change, the performance of the window can be electronically adjusted to suit conditions. A schematic illustrating how SageGlass ${ }^{\circledR}$ EC windows work is shown in Figure 1. In the clear, or un-tinted, state, the window is transparent to both the visible and the near infra-red radiation 
impinging on the window. This radiation is transmitted through the window into the building interior. The SageGlass ${ }^{\circledR}$ films can be tinted over the range of fully clear to fully colored, and held anywhere between these states. As the films are tinted, they become more absorbing, and depending on the level of tint, a fraction of the incident solar radiation is absorbed by the films. This reduces the amount of both visible and near-infra-red radiation transmitted through the window. The absorption of this energy causes the EC pane to heat up. As a result of the lowemissivity coatings, which are an intrinsic feature of the SageGlass ${ }^{\circledR}$ films, the heat built up in the EC pane is preferentially radiated back out of the building. This is shown schematically in Figure 1. Notice that the visible transmission never drops to zero, so a view to the outside is always maintained, and after all, this is the reason for putting in a window in the first place.

SageGlass ${ }^{\circledR}$ EC glazings offer the potential to save cooling and lighting costs, with the added benefit of improving thermal and visual comfort. Control over solar heat gain will also result in the use of reduced capacity HVAC equipment.

If a step change in the energy efficiency and performance of buildings is to be achieved, there is a clear need to bring electrochromic technology to the marketplace. This project addressed the need to accelerate the widespread introduction of EC windows into buildings and thus maximize the total energy savings in the US and worldwide.

The R\&D activities in this project will improve process and fabrication procedures for better device performance and higher yields. The goal is to reduce the product costs to the point necessary for broad penetration of architectural markets.

\section{EC Window Technology}

The SAGE device is a series of thin films deposited onto a glass substrate one on top of the other to form a functional EC stack. This is shown in Figure 2. The outermost layers are transparent conductors, which are used to apply a voltage to the active layers that are sandwiched between them. The active layers consist of an electrochromic (EC) layer, an ion conductor (IC) layer, and a counter electrode layer (CE). Charge, in the form of electrons and ions, is shuttled between the $\mathrm{CE}$ and the EC layers, producing the bleached and colored states respectively: The electrons are passed around the outer circuit, while the ions are transported through the IC. Insertion of charge into the EC layer will cause that layer to color to a depth that depends upon the amount of charge transferred. The effect is completely reversible, and is accomplished simply by reversing the polarity of the voltage. This is shown schematically in Figure 2. 


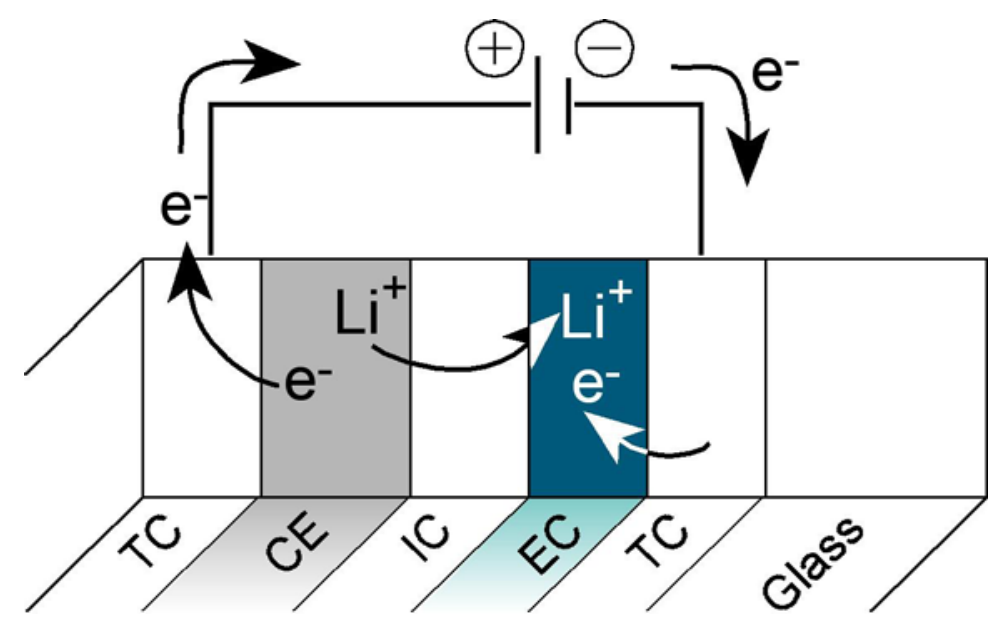

Figure 2 - The SageGlass ${ }^{\circledR}$ EC device showing the motion of the charged species around the 'circuit'.

\section{Significance of Problem}

The U.S. Department of Energy has estimated that the use of EC windows could reduce peak electric loads in buildings by $20-30 \% .^{\dagger}$ Similarly, energy calculations comparing SageGlass ${ }^{\circledR}$ EC windows with commercially available static glazings in the Southwestern U.S. result in average energy savings of $28 \%$ for a cooling dominated environment. These and other simulations indicate that significant cumulative energy savings in the U.S. would be achieved if current static glazings were replaced by dynamic EC windows in a significant portion of commercial and residential buildings.

EC windows can be lightened or darkened electrically to minimize solar heat gain and block glare while maintaining daylighting and a view through the window. They also significantly attenuate that part of the solar spectrum that causes fading of furniture and fabrics. This solidstate window functions with no moving parts and also provides security benefits since any break in the window can interrupt an electric circuit triggering an alarm.

To achieve significant national energy savings, the penetration of EC windows into existing markets must be maximized so that the largest cumulative energy reduction can be realized. The rapidity with which EC windows can be introduced and replace a current static glazing is a strong function of the cost. While the fundamental window performance requirements (e.g. optical quality, dynamic range, uniformity) could already be achieved, this proposal addressed the need to continually improve production efficiencies and throughput to achieve cost effective manufacturing (Tasks I, II, III). Equally important, the EC IGU's that are fabricated must be easily integrated into the existing products (skylights, vertical windows, doors, etc) of window companies. Task IV focused on EC window designs requiring minimal modifications of sashes/frames and/or extra tooling costs.

\footnotetext{
${ }^{\dagger}$ E.S. Lee et al. Energy Performance Analysis of Electrochromic Windows in New York Commercial Office Buildings, LBNL-50096, April 2002, Lawrence Berkeley National Laboratory, Berkeley, CA.
} 
The implementation of highly efficient processes is perhaps the most crucial hurdle and the source of greatest risk. Creative approaches to film deposition are required to minimize capital investment, and reduce the variability in coating properties over large areas. It is also essential to relentlessly attack and eliminate point defects in the EC films. Depending on the user application, a non-coloring spot $1 \mathrm{~mm}$ in diameter is not repairable and can cause rejection of the window. Finally, we must strive to remove any barriers to implementation of EC IGU's by the window companies. Consequently, the vision area (area of window covered by switchable films) must be maximized so that the non-coloring perimeter region is obscured by existing frames. Additionally, the wires, bus bars, and solder joints must be durable during window fabrication and over its life in the field. Wire routing schemes must be compatible with current hardware and take into account environmental conditions (rain, UV exposure, etc).

This report discusses the activities carried out to achieve these goals. 


\section{Report on Workplan Activities}

The following is a detailed description of the technical progress achieved. .

\section{Improved EC Layer Deposition Process}

The EC layer is currently fabricated at a slightly elevated temperature. In order to improve both performance and manufacturability, it is desirable to reduce the deposition temperature, without affecting any of the physical properties of the film in an adverse way. One approach that has been studied during this project is to replace the thermal energy with energy supplied directly to the growing film by means of plasma bombardment. Lawrence Berkeley National laboratories (LBNL) are world-renowned experts in plasma applications, and have both plasma source

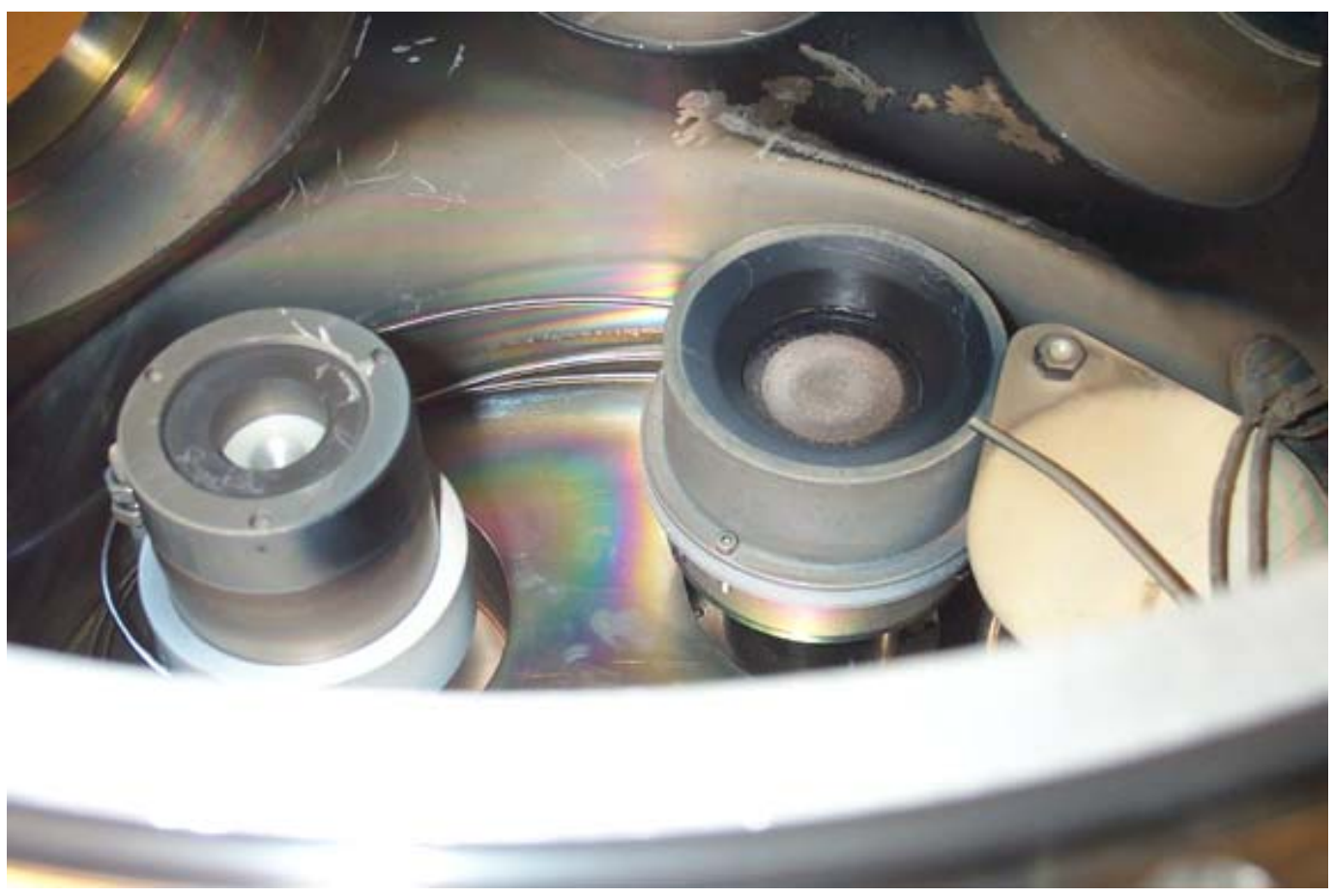

Figure 3 - Initial test setup for room-temperature deposition of the EC layer: left: single-hole Constricted Plasma Source (CPS), right: magnetron with metallic-target. Sputtering is done in sputter up configuration with glass substrate adjustable in lateral and vertical position. No heater was installed in this setup.

manufacturing capabilities as well as film deposition technology. For these reasons, they were chosen as partners for this part of the project.

1) The following sections (I.1 to I.3) detail the work done jointly between SAGE and LBNL in this area. The project begins by depositing 'baseline' EC layers at LBNL. These are simply thin films similar to those produced at SAGE, thereby providing a reference point for subsequent plasma enhanced depositions. The LBNL films are used to produce small area EC devices by carrying out subsequent film depositions using the production equipment at SAGE. Next, plasma enhanced films were deposited at LBNL, and they too 
were used for EC device production and their performance compared to the performance of the 'baseline' samples. Some details of the development of various plasma sources carried out during this work is also discussed.

\section{I.1 Deposit of Baseline and Ion Assisted EC Layers}

At the beginning of the project, LBNL adopted a high-risk, high-payoff approach in using an existing plasma source known as a constricted plasma source (CPS), for assisting roomtemperature sputter deposition. The plasma source was of the single-hole constricted-glow type, which was developed and manufactured at LBNL in the late 1990s. The source is able generate plasma flow containing a mixture of neutral gas molecules and atoms, excited molecules and

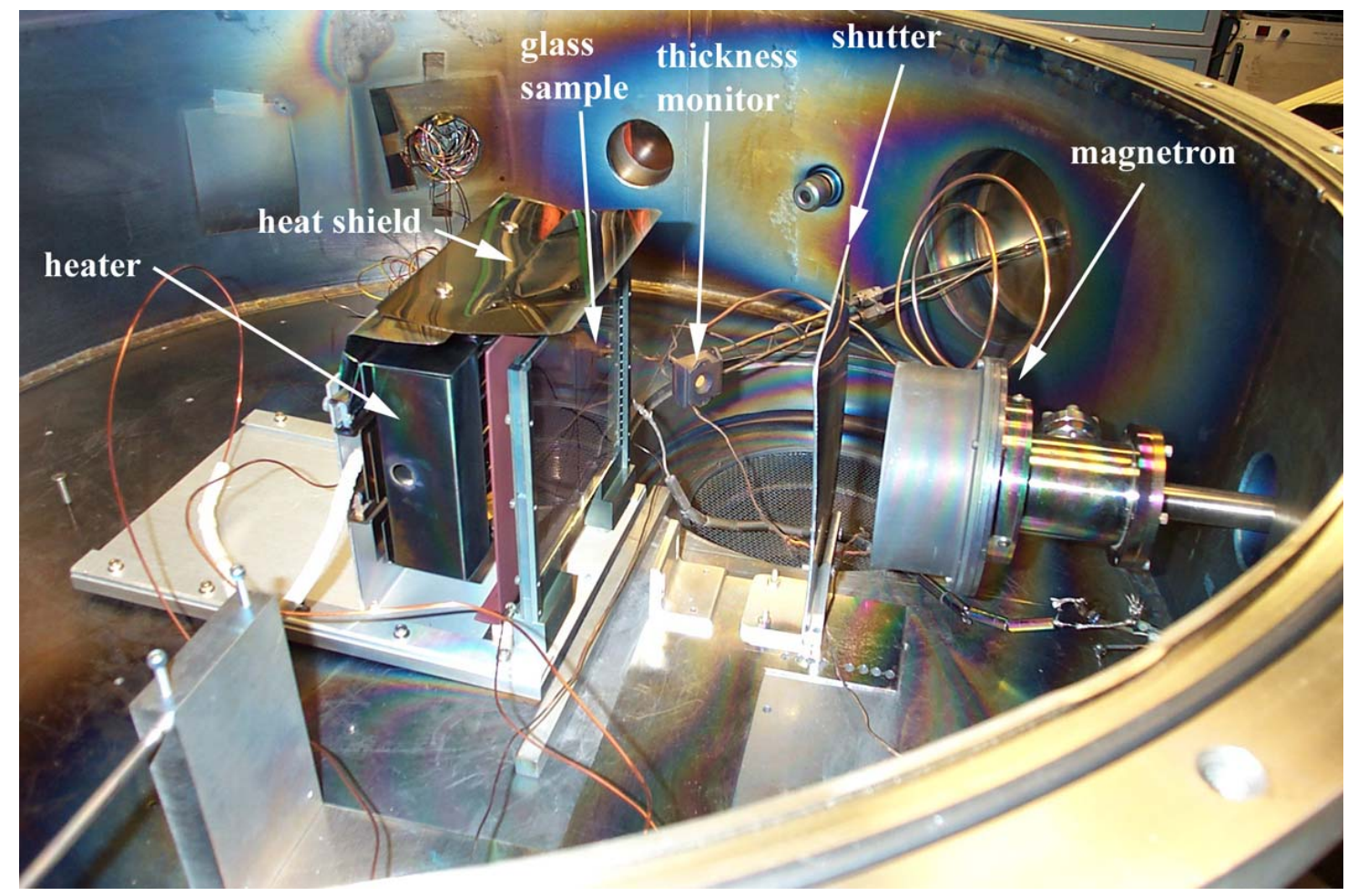

Figure 4 - The second setup with vertical sputtering and heater incorporated. This shows the heater with the copper thermal diffuser located behind the sample holder.

atoms, as well as ions and electrons. The total flux from a single-hole source is less than $1 \mathrm{~mA}$, and rapid decay with increasing distance is well known. Still, a possible positive effect on EC layer growth would have been a breakthrough towards low-temperature deposition. The setup used is shown in Figure 3.

Two sets of samples with the EC layer on coated glass substrates were produced with this setup. Samples were shipped to SAGE for making complete EC devices. The performance of the devices was found to be poor, showing that the simple setup did not give the desired results. Based on this intermediate result, three tasks were pursued in line with the research plan:

1. Implementation of a substrate heater with the goal to reproduce high-temperature-quality EC films at Berkeley Lab such that they could be used as a reference point. With this 
reference point in place, plasma assistance could then be applied in a more systematic manner to reduce deposition temperatures lower than conventional.

2. Development of a new plasma source that is capable of delivering a much higher intensity of plasma flux yet having ions at relatively low energy to avoid ion-induced damage to material's structure.

3. High resolution material analysis of well-performing and less-well performing material to better understand what constitutes "good" material in the sense of SAGE devices.

In order to achieve these aims, a new deposition system was set up in which a radiative heater was incorporated in a different, upgraded vacuum chamber. The shape and port locations of this

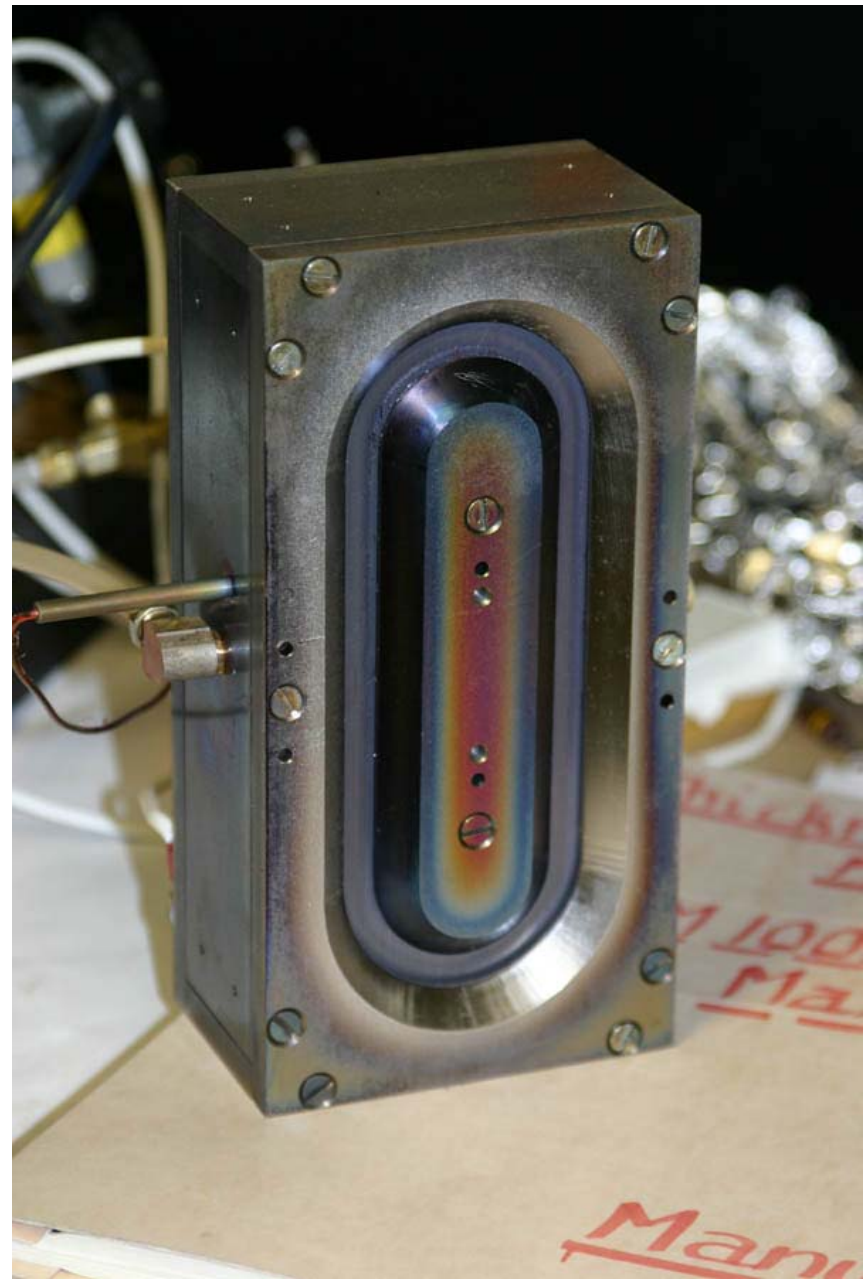

Figure 5 - LBNL-made closed-drift anode layer plasma source. chamber dictated vertical sputtering, which is similarly suited to electrochromic coatings as is the sputter-up configuration: both systems are designed to minimize particulates in the film.

Significant effort went into the design and set-up of the heater. Initial tests with a radiative heater, which illuminated the glass substrate directly, led to several incidents where the glass was broken as a result of too great a thermal shock. Installation of a copper plate between the heater and the substrate allowed indirect heating of the substrate, thereby largely eliminating breakages.

An additional issue which is almost always present is the measurement of the actual substrate temperature. This is because the glass is relatively transparent, so some of the higher wavelength radiative heat can pass straight through the sample. In contrast, a thermocouple is often more absorbing than the glass at these wavelengths, so can give a substantially higher measurement then the actual temperature. The usual solution for glass is to use a method utilizing the emitted IR from the glass, such as a pyrometer. The necessity to use a copper plate as a heat transfer mechanism made this very difficult, so in this case, the temperature of the substrate was determined by measuring the temperature of the holder using a thermocouple. Some experiments were carried out to 'calibrate' the measured temperature with the actual temperature, and so an approximate temperature was obtained. 


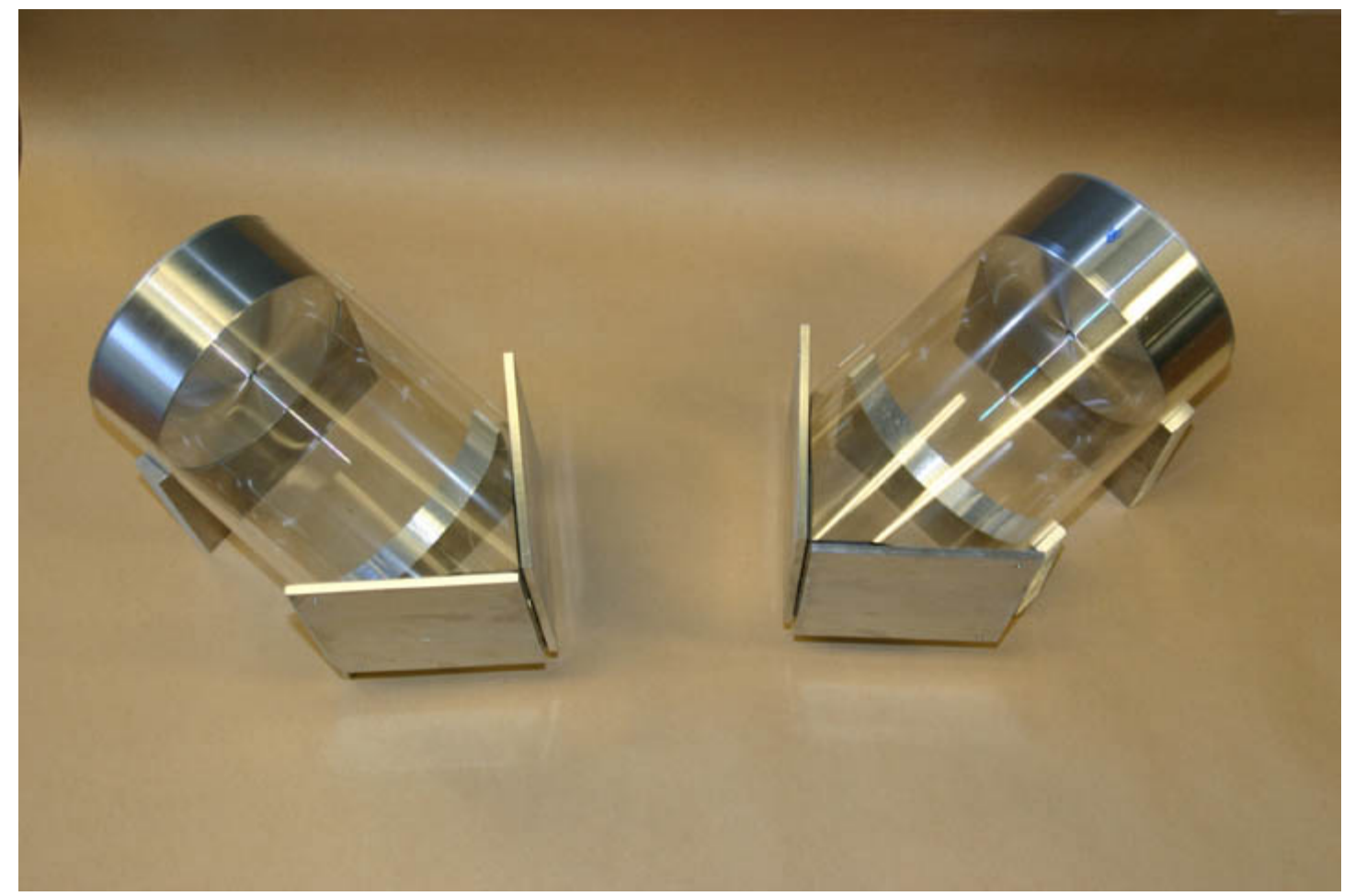

Figure 6 - Two novel plasma sources, not yet completed, for future plasma-assisted deposition. The size of the each source body is 5" in diameter; hence the plasma should have an appropriate size matched to our test samples. Note that the output shape fort the plasma is a 5" long slot, rather than a hole.

With the new system, EC films were deposited at elevated temperature and at various sputter conditions. After close consultation between LBNL and SAGE personnel, conditions were identified which resulted in films that served as a reference point for plasma-assisted deposition.

In order to make plasma-assisted deposition work for this application, suitable plasma sources need to be developed and incorporated. The original CPS did not provide enough flux to make any significant improvement, which is in part due to large distance between source and film. In the original configuration, this distance was large as to not block the flux of sputtered metal from the magnetron to the glass sample.

Two developments were pursued in an attempt to improve matters. One was a closed-drift anode layer source. The concept of such source dates back to the Soviet space program of the 1960s, and in recent years some companies have adopted the technology for glass coating and plasma assistance. Therefore it seems reasonable to test such a source for the present application. No low-cost source is on the market, and therefore a simple test system was built at LBNL and is shown in Figure 5.

A 6-inch long closed-drift anode-layer plasma source was designed and manufactured; it is suitable for test but not for extended runs since no water-cooling was incorporated for cost reasons. This type of source can easily be scaled to any arbitrary length, as later needed for large area coating. It was found that the source could operate in basic modes which could be obtained by adjusting the gas flow. Preliminary tests indicated that the source delivered a flux of ions that exceeded the CPS by over a factor ten. Unfortunately, higher-energy ions were possibly 
involved which may have caused ion damage to the growing film and also contamination of the film by introducing iron atoms sputtered from the cathode-housing.

For these reasons, this particular source was not used during this work, but future work could focus on addressing the issues raised here.

Following that approach, another source concept was pursued. It was based on previous CPS research, and brought the CPS to a new level in terms of output and scaling. A new shape of the source was designed accommodating the presence of a magnetron while minimizing the distance between source and glass substrate. For uniformity and symmetry reasons, plasma should come from two sides of the magnetron, and therefore two sources of the same design were built and are shown in Figure 6.

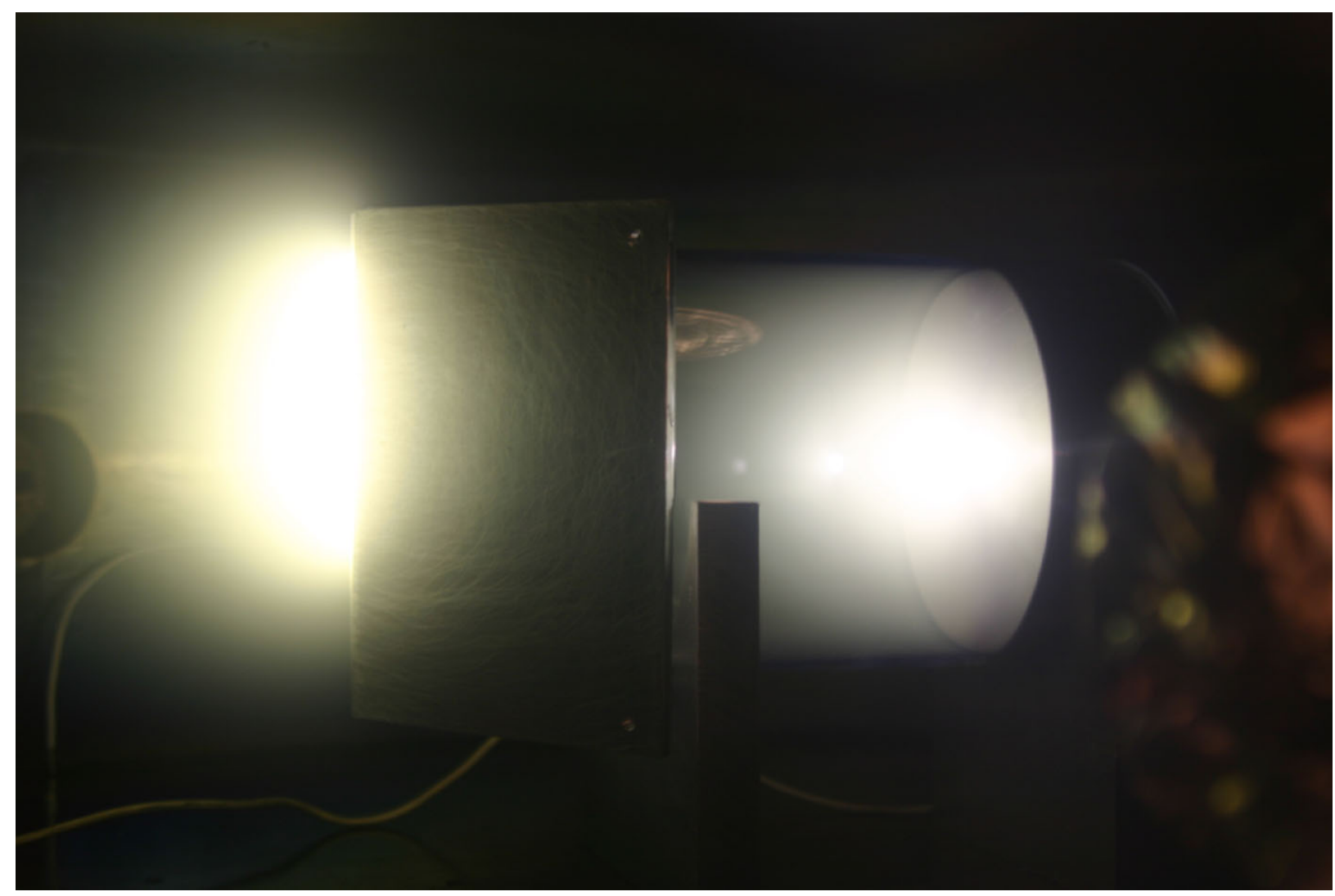

Figure 7 - First test of new gas plasma source operating with oxygen. The left (greenish) light is the useable plasma flow produced at pressures compatible with magnetron sputtering.

The new linear (slit) plasma source of the Constricted Glow Discharge type was tested and can be seen in Figure 7. Distinctly different operational modes were discovered, leading to greatly enhanced output compared to previous versions of the source; outputs, as measured in terms of ion flux, can be enhanced by about 400\%-1000\%. The three photos (Figure 8, Figure 9 and Figure 10) illustrate the performance: 


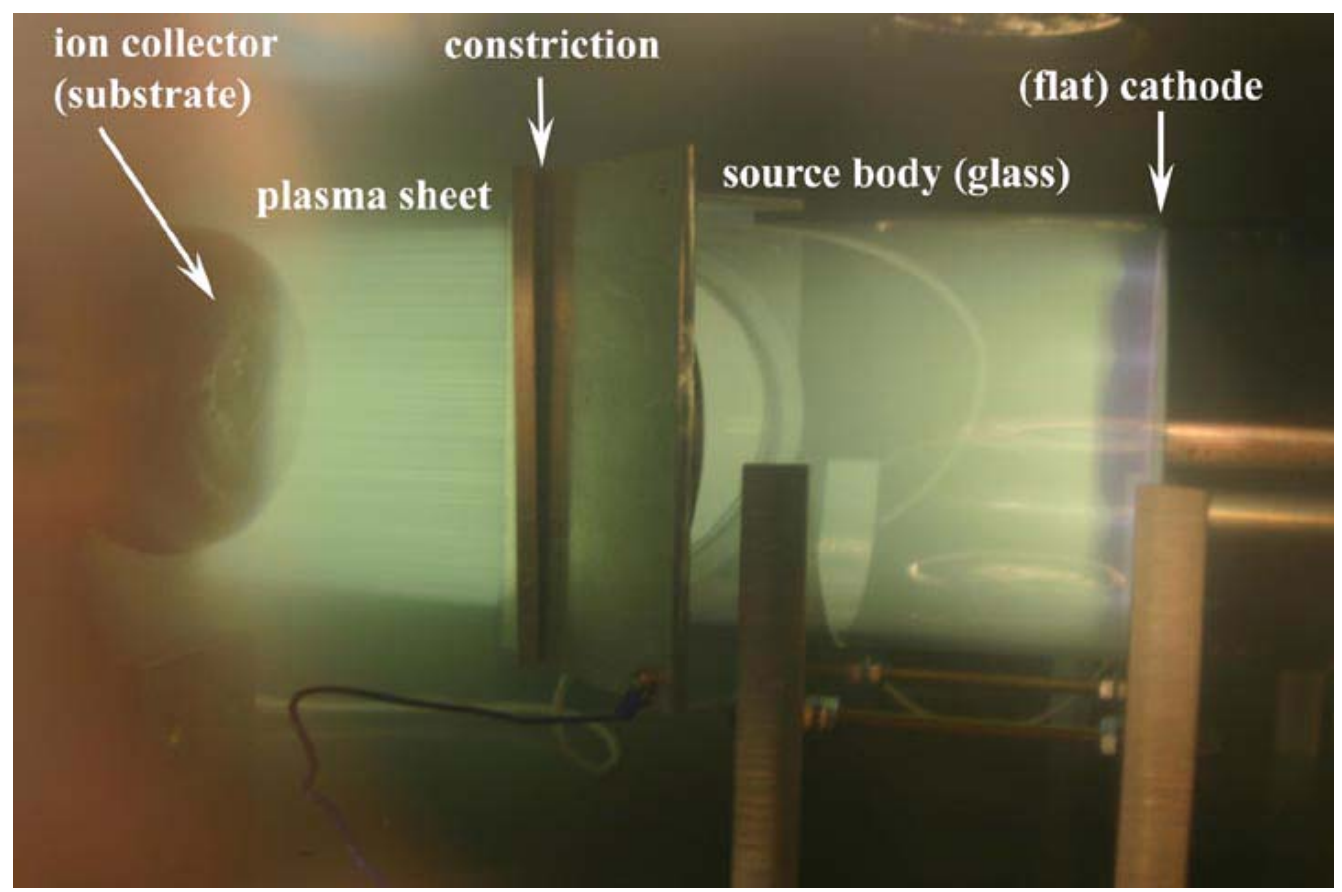

Figure 8 - This photo shows the source in normal operation; the cathode is to the right, the main plasma inside the source (glass body) appears semi-transparent, and the usable plasma is to the left, impacting the ion collector (corresponding to substrate in a coating setup).

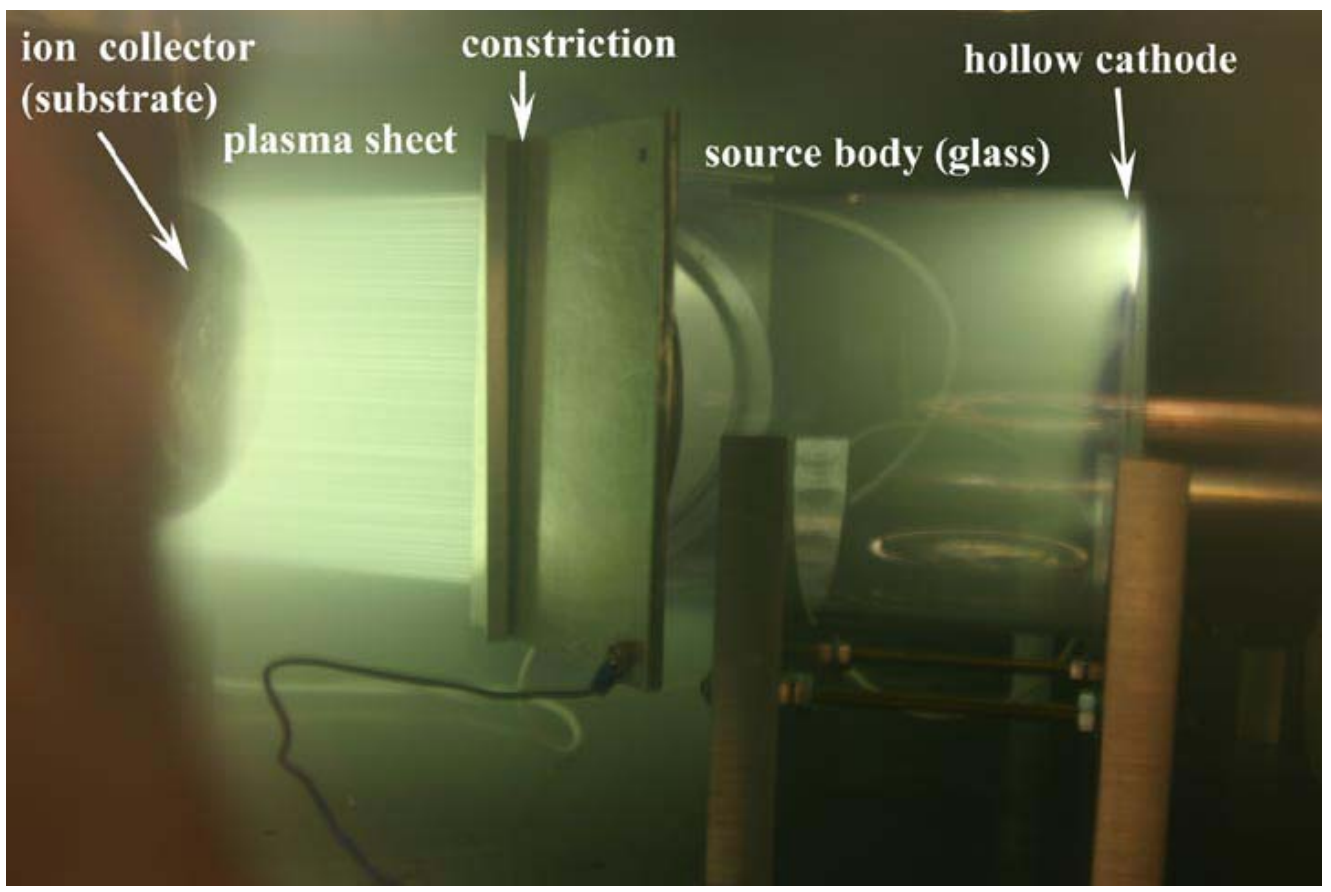

Figure 9 - When the cathode is made with holes of suitable size, electron multiplication can occur through the hollow cathode effect. The hollow cathode switch-on is related to a feedback effect and appears therefore as a distinct switch-on. The usable plasma is enhanced by about $400 \%$. 


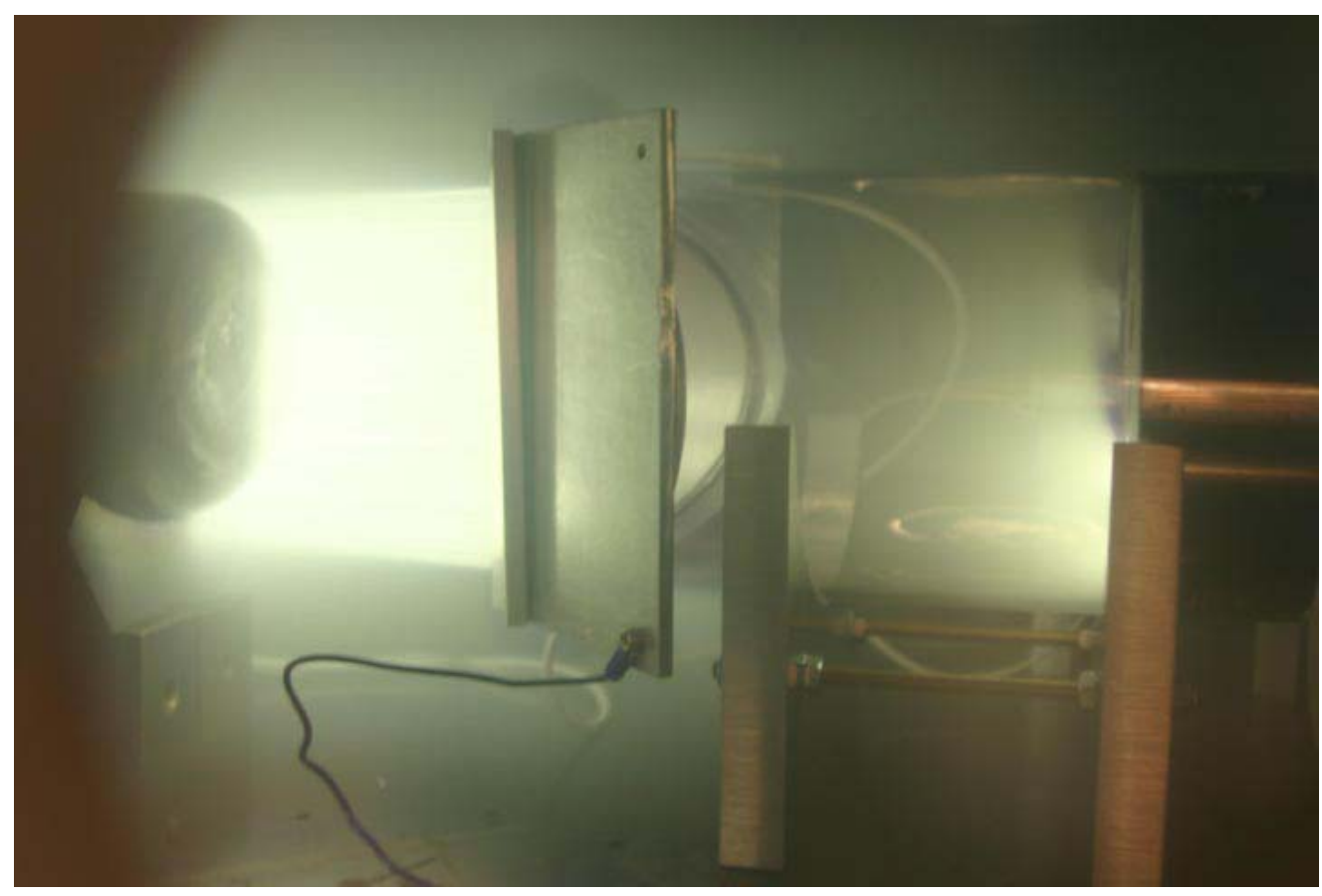

Figure 10 - When the discharge current is increased, the source may switch in a high power mode, and the useable plasma is increased by about $1000 \%$ compared to the original "normal" mode.

The discovery of these enhanced modes may represent a breakthrough in the development of the Constricted Plasma Source as a high performance source. The output is greatly enhanced and the plasma composition is changed, most likely from molecular to atomic, which is of great importance to film growth. However, the high power modes are not yet stable, and no diagnostics have been done. Most importantly, arcing at cathode, plasma oscillations, overheating of cathode and constricting slot and other components have to be addressed.

Unfortunately, time constraints dictated using the source in its low-power mode, which is stable, in order to make samples for this work. The further investigation of the higher power modes offers an interesting future development.

The equipment described above was used to produce both baseline - using the heater to provide energy to the growing film - or plasma assisted - using the ion source instead of the heater - EC films. These were then subjected to a number of characterization experiments to determine whether there were any significant differences between the two approaches.

\section{I.2 Characterization of Baseline and Ion Assisted EC films}

Of great interest is the structure and composition of the films as well as details of film interfaces. The preliminary characterization used X-ray diffraction (XRD) to determine the crystalline structure of the films. Films deposited at LBNL using the original setup showed no crystalline structure, other than the underlying transparent conductive film. Samples made at SAGE at elevated temperature indicated crystalline structure evident, as did samples made at LBNL at elevated temperature. 
X-ray diffraction was used to analyze a number of EC samples, made at LBNL and SAGE. As can be seen in example below, (Figure 11) the main feature is to be found at about $2 \theta=23$ degrees.

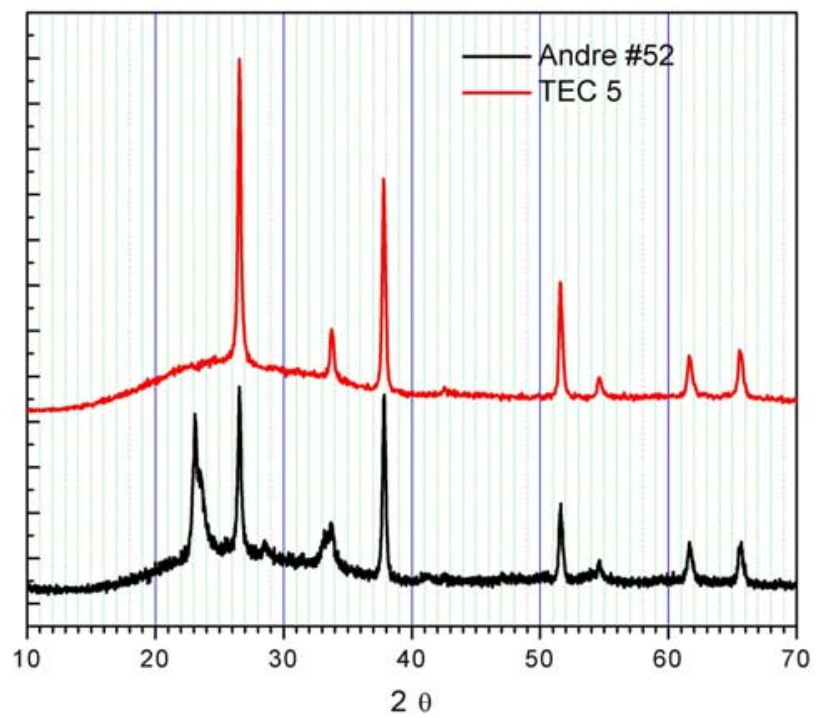

Figure 11 - X-ray diffraction pattern for LBNL-made EC film, deposited at about $250^{\circ} \mathrm{C}$ (conservative thermocouple measurement, actual temperature could be somewhat higher). For comparison, the upper curve shows the peaks due to the TEC coating only.

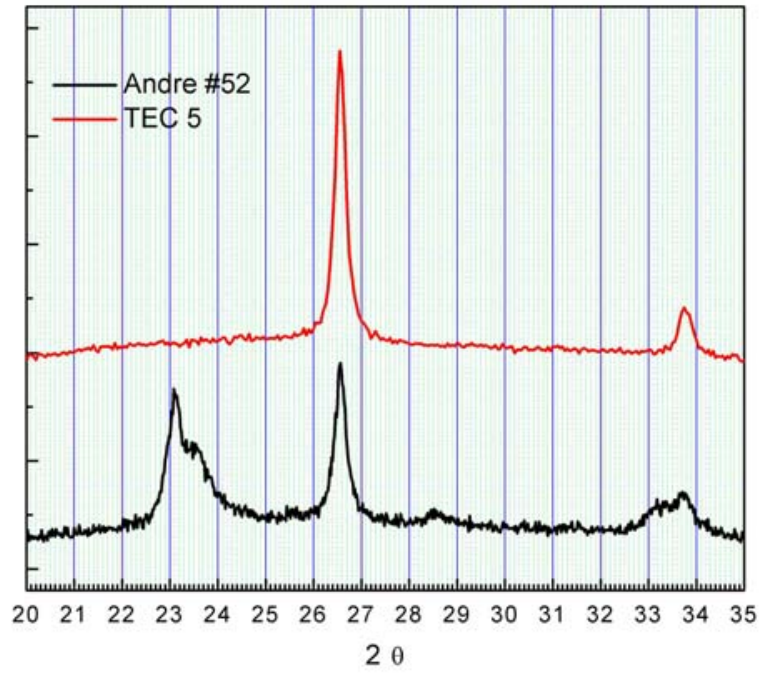

Figure 12 - The same data as shown in Figure 11, but with higher resolution. 
For comparison, Figure 13 shows a SAGE film.

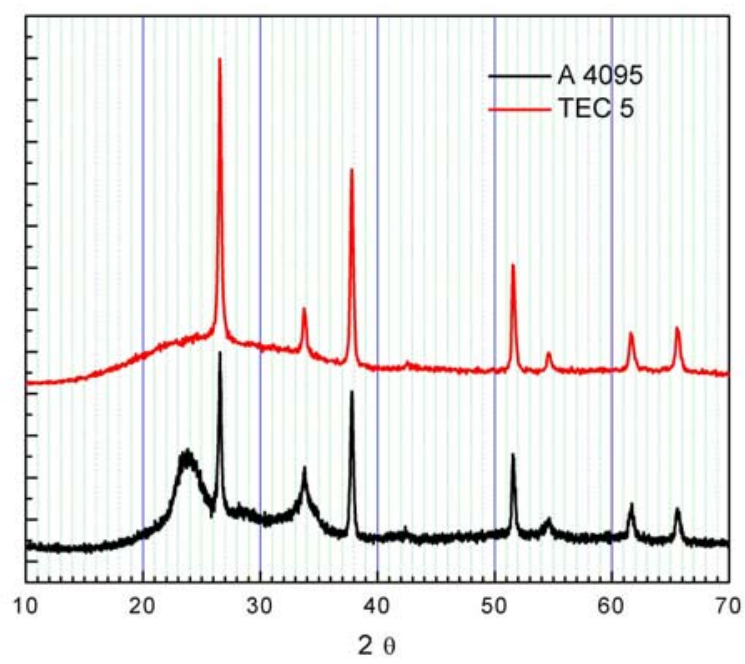

Figure 13 - EC film made by SAGE's process. There are subtle differences in the shape of the relevant peaks when compared to Figure 11 and Figure 12, indicating a difference in crystalline structure.
The findings by XRD can be summarized as follows:

1. Samples prepared at room temperature are amorphous.

2. Comparing the samples prepared at LBNL with the ones provided by SAGE, it appears that LBNL samples are oxygen deficient.

3. The difference in the pattern between samples of different electrochromic behavior seems to be too subtle to be detected by XRD.

As a result, TEM studies were undertaken. The microstructure of a standard (SAGE produced) EC coating on TEC glass was studied using Transmission Electron Microscopy at LBNL. The primary aim of this study was to provide an insight into the microstructure of a standard coating, and therefore allow a comparison to be drawn with future EC coatings fabricated using ion beam assistance.

Cross sectional TEM (X-TEM) samples were prepared from the 4mm thick samples provided. These were initially cut in $5 \mathrm{~mm}$ strips and the coated sides were glued face to face as shown in Figure 14(a). Small slices of approximately $0.5 \mathrm{~mm}$ were the sectioned out using a diamond saw, and $3 \mathrm{~mm}$ discs were cut out aligning the region of interest in the middle as shown in Figure

14(b). The
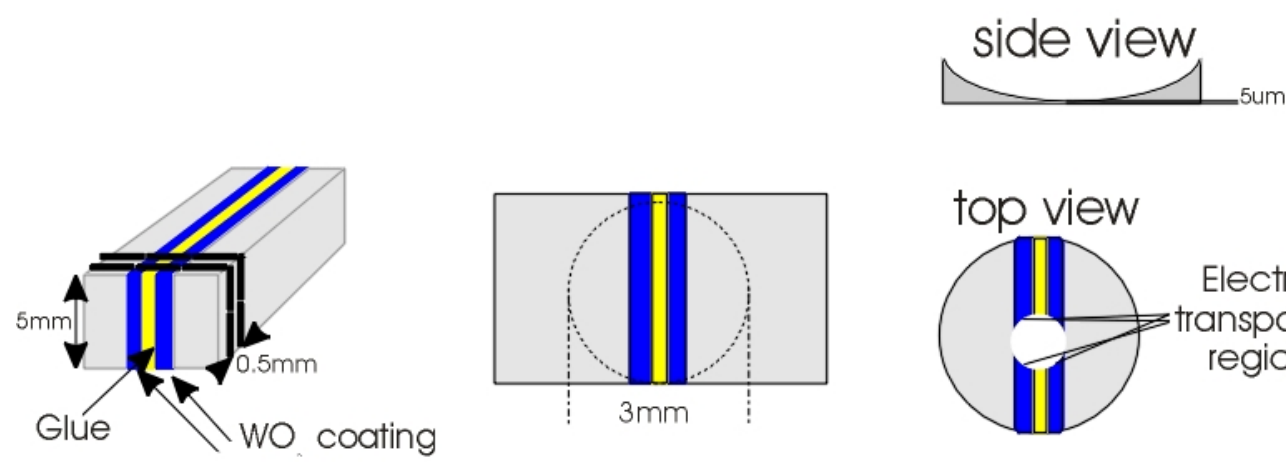

(a)

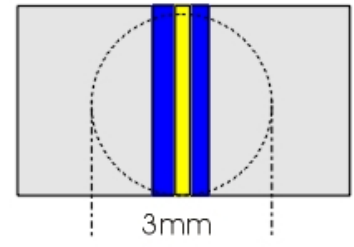

(b)

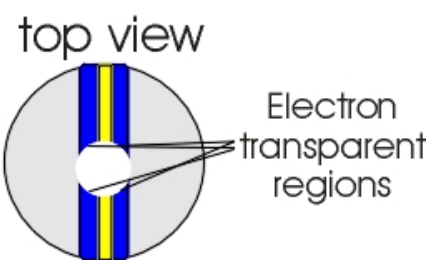

(c)

Figure 14 - Schematic of X-TEM preparation. Note: the figure is not to scale.

sample was then dimple ground mechanically to approximately $5 \mu \mathrm{m}$ in the middle using a dimplier. Final thinning was done using a Focused Ion Beam until a hole was 
thinned out which gave electron transparent regions near the edge of the hole (see Figure 14(c) top view).

A preliminary image of the coating with the substrate is shown in Figure 15(a) with a corresponding diffraction pattern (Figure 15(b)). The Diffraction pattern of this standard SAGE production sample shows that the coating is more polycrystalline than some of the samples that were prepared at LBNL using plasma assistance.

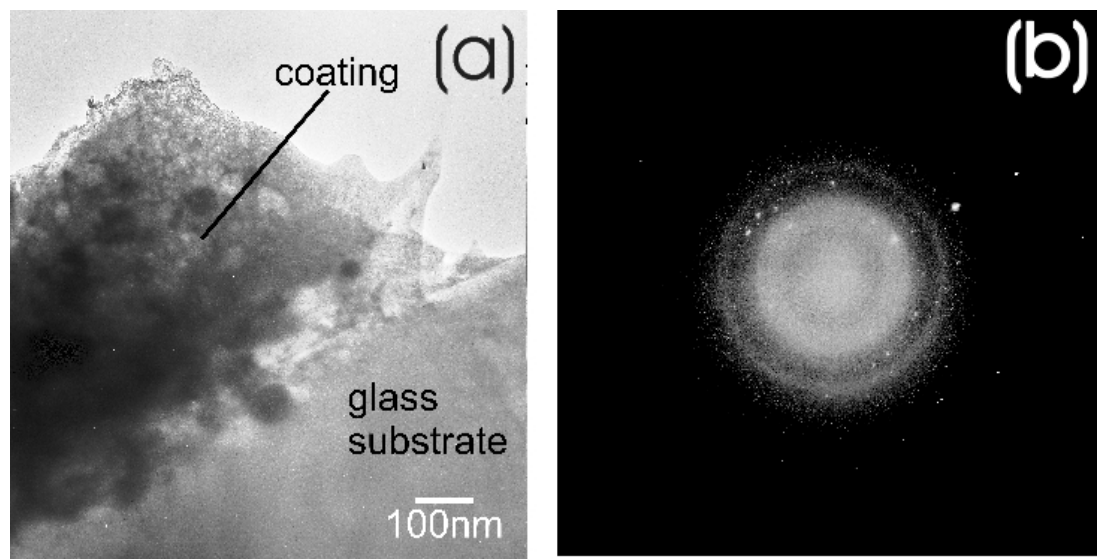

Figure 15 - TEM image of coating and its corresponding diffraction pattern.
The study suggested that there were differences in the crystallinity of films prepared by the 'conventional' approach when compared with films prepared using plasma assistance. It is conceivable that this may have some effect upon the performance of the coating, however, bearing in mind it is possible to produce electrochromic behavior from totally amorphous films, and

this is unlikely to be the main determinant of performance. Unfortunately, a systematic study of the EC layer material as a function of processing parameters was not possible using this technique due to the prohibitive cost.

\section{I.3 Fabricate LBNL films into small devices}

Several runs of films deposited at LBNL were made into devices at SAGE. This is a fairly simple process, although care had to be taken to avoid exposing the films to anything that could compromise the behavior of the device. Films made at LBNL on the same substrate type as used by SAGE are transported back to SAGE for device completion. The partially completed devices have the remaining films deposited using the standard process, resulting in functional EC devices. The performance of these can be compared with control devices run in parallel, to determine the effects of the plasma treatment or other experimental variations.

A first round of test samples was made at LBNL to determine the necessary $\mathrm{Ar} / \mathrm{O}_{2}$ flow rates in the new chamber configuration leading to EC films that are approximately stoichiometric. The deposition rate was calibrated by determining thickness with a profilometer. These samples were sent for completion into devices at SAGE.

The performance was generally poor, with the main failure characteristics being: low initial transmission, poor dynamic range, and high leakage current. Even in the one sample which colored 'reasonably' i.e. to around $8 \%$, there was a significant amount of loss of transmission upon cycling. (Note that the control samples performed satisfactorily). Basically, the behavior of these samples can be explained by the low level of blindcharge ${ }^{\ddagger}$ in the EC layer. It is noted

\footnotetext{
‡ M.S.Burdis and J.R.Siddle, Thin Solid Films 237, (1994) 320.
} 
that the SAGE process had been tuned over the last few years to accommodate the EC layer produced on the Pilot Line, and this is deposited in a highly oxygen rich plasma. The samples produced by LBNL were produced in a highly argon rich plasma, which is likely to be the main reason for the poor performance.

Unfortunately, the deposition chamber at LBNL was susceptible to the well-known problem of the disappearing anode when running in a highly oxygen-rich atmosphere. This means that stable and repeatable operation was difficult, and so it was decided to modify the equipment slightly to incorporate a second cathode, and deposit films using AC sputtering. This circumvented the problem, and allowed rapid deposition of films at the required gas concentrations.

A set of new samples was coated using enhanced oxygen flow, compared to previous runs, and variety of settings of other parameters with the goal of producing high temperature samples that can be used as a control for low temperature plasma-assisted deposition. The samples were shipped to SAGE for evaluation. Preliminary results were encouraging with the devices at least showing some coloration over a reasonable range.

Issues remained with uniformity and so a substrate motion stage was constructed, with the aim of moving the substrate through the plasma in one axis, thereby smoothing out the uniformity in this axis to some extent.

The next set of samples made by LBNL, with plasma assistance was processed into complete devices, and the initial characterization completed. None of the devices got to the point where it could be considered to have passed the SAGE specification. One device showed reasonable performance - the dynamic range being from $5.7 \%$ up to just over $60 \%$. This set of samples gave very similar performance to the 'control' samples made previously using conventional thermal heating, and as such represents an improvement over the control samples, in that the current group was processed without preheating. The only additional energy came from the plasma treatment and the sputtering plasma.

One remaining question to be answered is whether the plasma treatment can be used to significantly reduce the heat treatment required in the large area process, and thereby give rise to substantial savings in hardware and process time. Another issue was whether the plasma exposure was simply heating the glass substrate rather than just the thin films. This would mean that the plasma treatment was simply mimicking the conventional thermal process, rather than providing energy to the growing film alone. One of the goals of the program was to be able to deposit the EC layer at a reduced temperature in order to make it suitable for deposition onto low-temperature substrates such as plastic.

This work showed that it is possible to produce an electrochromic layer using plasma assistance, rather than conventional thermal heating. Unfortunately, the problems associated with transporting the samples between different vacuum systems, as well as the non-uniformity of the films produced in the development system, albeit after significant improvements made during the work, made it difficult to investigate the subtleties which must be understood in order to make a commercially viable process. It is probably necessary to develop a fully integrated plasma assisted deposition system within the production equipment to further understand the possibilities and pitfalls of using plasma assistance to reduce or replace the conventional heating step within the current process. 


\section{Thin Film Capping Layer}

It is important to protect EC films from handling and environmental damage during processing steps in which the films are exposed to ambient conditions. After the top layer of the EC device is deposited, the device is removed from the vacuum chamber and can be exposed for as long as 24 hours during testing, shipping and IGU fabrication. During this time, the layers are fairly sensitive to moisture and other contaminants. Therefore utmost care must be taken to protect the films prior to sealing in the standard desiccated IGU.

A capping layer that retards the diffusion of contaminant species into the EC device would reduce yield losses due to non-uniform coloration. In earlier work, it was found that a proprietary layer applied over the top transparent conductor gave an indication that it may be capable of retarding EC device degradation. Unfortunately, this proprietary deposition method was not easily compatible with the in-line sputtering system required for cost-effective EC device production. It was therefore proposed to develop an optimized - in terms of film density and barrier properties - capping layer that could be deposited in a cost effective way. As an added benefit, a capping layer of the relevant thickness can serve as an antireflection coating and thus increase the clear state transmission of the EC device by a few percent.

The following sections describe the work done on this task, beginning with basic film property measurement, and continuing with some prototype device fabrication.

\section{II.1 Install Target, Measure Film Properties}

A rotatable target of a suitable material was obtained, mounted onto a rotary cathode, and installed into the Pilot Line Coater. This was later transferred to the Production Coater, and ultimately replaced with a dual rotatable which uses an AC power source.

Process conditions for capping layer deposition were surveyed to find the processing limits for operating the target in oxide mode rather than metal mode. A high rate process is desired in order to yield a high density film. A factorial experiment was conducted to determine the effects of power, oxygen flow rate and total pressure on the thin film deposition rate.

Deposition conditions of highest power, lowest oxygen flow rate and highest pressure yielded the fastest deposition rate. This rate was approximately double that of the current standard deposition conditions. All films were found to be transparent with no observable absorption. Additionally, all films were found to have no influence on the overall device behavior upon subsequent heat treatment, other than yielding an improvement in the overall transmission as a result of the antireflective nature of the film.

Preliminary statistical analysis revealed that all three factors were significant, in addition to a significant interaction effect for power and pressure. Overall, the statistical model predicts that higher power, higher pressure, and lower oxygen flow yield higher deposition rates.

\section{II.2 Fabricate prototype EC devices}

Devices were run with the capping layer deposited at a standard thickness of approximately one quarter wave, prior to venting and final heat treatment. The resulting devices were indistinguishable from those processed either immediately before or immediately afterwards, apart from the optical differences expected due to the capping layer, indicating the presence of 
the capping layer has little effect on the electrical properties of the EC device. This has had positive implications for process flow in the production facility.

A design of experiments (DOE) run was carried out to assess the potential advantages of a capping layer with a higher refractive index but potential to yield a better diffusion barrier, over a 'standard' one. The higher index layer leads to devices with higher reflection than bare devices, or devices with a lower index capping layer. This leads to a lower transmission, but it is possible to redress this by deposition of a low index layer onto the higher index capping layer. It is convenient to label the possible layers as L (low index), $\mathrm{H}$ (higher index) and HL (the relevant combination). EC devices were therefore fabricated with the following capping layers: (i) L, (ii) $\mathrm{H}$, (iii) HL, and (iv) samples with no capping layer to act as controls. Two groups of devices were treated with a variety of capping layers, each designed to give a reasonable thickness - to maximize the moisture blocking potential - but maintain the highest possible bleached state transmission for each configuration by optimizing the optical thickness' for the layers. Each group contained the same range of samples, i.e. two with no capping layer, and six other devices with three different capping layer arrangements.

Half of these samples were being cycled in a modest humidity, while the others were stored as controls for further experimentation later. The object of the experiment was to determine the degree to which the capping layers influenced device degradation.

Figure 16 shows the initial transmission measured for the samples made with different capping layers, showing the excellent repeatability for the replicates within the experiments, as well as the expected trend for the different capping layers. It is seen that the $\mathrm{H}$ devices have the lowest transmission, as expected, while those with the L top coat have the highest. Uncoated devices are midway between these two.

The relevant performance parameters, such as transmission, current, uniformity, occurrence of defects, etc., was tracked as the devices were cycled. 
DESIGN-EXPERT Plot

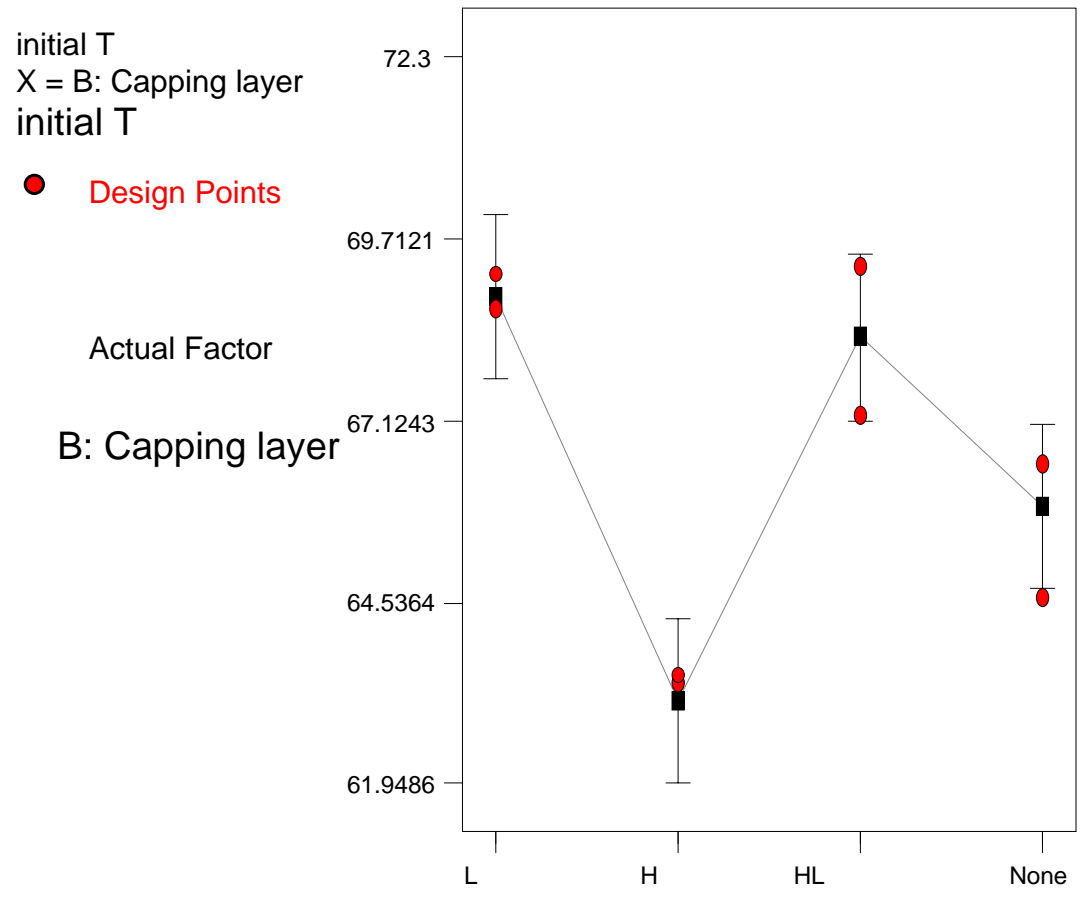

The devices were stressed in humidity for over 1500 fully colored to fully bleached cycles. All showed signs of performance degradation, indicated by areas of permanent blue coloration, reduced dynamic range, slower switching rates and higher leakage currents. The leakage currents are all increased, irrespective of the presence of a capping layer. The dark state transmission appeared to increase and then reach a saturation level, suggestive of an equilibrium state being achieved. Switching rates all decreased during the duration of the experiment, with no apparent sign of reaching an equilibrium saturation level.

There were very few signs that the capping layers affected the changes seen in the devices. However, it appeared that the samples with capping layers that were fired at elevated temperatures appeared to take longer to reach the saturation value. This may be a possible observation of slower diffusion of the contaminant (assumed to be water) through the heattreated capping layers. Further analysis is required to completely understand this.

A second set of monolithic substrates was stored in a dry environment since manufacture in early November 2004. One reason for this was to check the effects of storage on devices, and compare the properties with the devices which have been continually cycled. The second reason is to evaluate the short term benefits of the capping layer to see if there are any at all.

The capping layer did not provide any benefit to the devices which were stored. Each of the devices in this group lost dynamic range and became somewhat slower to switch, but to a lesser extent than the devices which had been cycled. No difference was detectable between the devices with or without the capping layers. 
In conclusion, the overall objective of creating a capping layer which could be applied as part of an in-line process was achieved. This has had the benefit of improving the overall transmission in the bleached state for EC devices, by virtue of reducing the overall reflection. Unfortunately, it was not possible to demonstrate any benefit as a diffusion barrier for these layers.

\section{Defect Elimination}

The work in this task is aimed at elimination or reduction of two major classes of defects seen in the EC devices made at SAGE. The first, dealt with in Section III.1 to Section III.6 is referred to as a non-coloring dot. These are typically very small (of the order of $200 \mu \mathrm{m}$ in diameter) but can be visually objectionable. The investigations into their controlled generation and methods for elimination are discussed below. The generation of these defects has been fairly conclusively linked to the tempering operation, so the report begins with a discussion of the work done to understand the effect of tempering on the glass, and the factors which could be responsible for variations in dot size and density. A method for controllably generating the defect is then described, and this has been used to devise methods for eliminating or reducing their occurrence. Subsequent sections describe a variety of techniques devised to possibly eliminate or reduce the effect of the defects.

The second type of defect is referred to as a short. This occurs when the integrity of the device stack is compromised at a point, and the electronic current can flow unimpeded through the device. This leads to a lighter circular area, which can be up to $1 \mathrm{~cm}$ in diameter. Methods for elimination of these defects are discussed in Sections III.7 to III.9.

\section{III.1.1 Survey and Optimize Tempering Factors}

Continuous improvements are necessary to reduce defects that substantially lower production yields by marring the visual quality of EC coatings with areas that fail to color. A diffusion barrier deposited on the substrate glass by the manufacturer is prone to mechanical damage, such as scratches and cracks. These cracks, although small enough to be acceptable for normal window glass production, have been shown to produce sodium contamination of the EC device, and are clearly linked to the observation of the non-coloring defect. The cracks are typically microscopic breaches that allow sodium to penetrate through the diffusion barrier into the growing EC film stack, particularly during processing at elevated temperatures. These sodium ions can subsequently diffuse radially, poisoning deposited films to form non-coloring dots, thereby resulting in an almost perfectly circular defect. This root cause has been confirmed via SEM/EDS analysis showing elevated sodium levels in the defect area and this theory further validated by experiments involving deliberately damaging the substrate surface under controlled conditions.

One mechanism known to facilitate sodium migration in glass is heat. A review of the historical data revealed a strong correlation between these defects and tempering, a process that typically raises the substrate temperature to near $700^{\circ} \mathrm{C}$. A survey of tempering factors was needed to determine the main variables responsible followed by optimization experiments to minimize their impact.

The process of tempering is relatively simple to understand. The aim of tempering is to increase the impact resistance of a piece of glass. This is done by introducing a compressive stress into the surface of the glass, which has the effect of reducing the ability of a crack to form and 
propagate. This is achieved by raising the temperature of the glass to around $700{ }^{\circ} \mathrm{C}$ in an oven, and then rapidly cooling it by blowing huge volumes of cool air onto it. This is a process known as quenching, and in the SAGE tempering furnace, the quench air is forced onto the glass from above and below simultaneously through a delivery system with tubes approximately $2 \mathrm{~m}$ in diameter to allow for the massive volumes required.

Tempering was initially done by an outside vendor, which required periodically taking a 'batch' of substrates to their facility. Several steps were involved in processing substrates before tempering, including some that have the potential to inflict damage on the surface. Historical data for the density of these dots were sorted according to two categorical factors (tempering lot and substrate preparation lot) and a single tempering factor (substrate residence time in the tempering oven). The data were then subjected to analysis of variance and $t$-tests, which revealed that all three factors had significant effects; the most significant of these was tempering lot. However, a follow-up designed experiment comparing long and short residence times in the tempering oven failed to reveal any substantial difference in the dot densities for the two groups or the surface stress that was thought to play an important role.

A limited set of key parameters was then identified for subsequent study, including residence time; quench airflow parameters, gas burner blower settings, and the transition speed of the glass from the furnace into the quench area. At the same time it became clear that the method used to determine dot densities on finished devices (manually counting defects in small, randomly selected areas) was inadequate and was therefore replaced with a semi-automated system employing a digital camera that sampled an area more than 25 times larger and reduced the signal to noise ratio by a factor of five. Additionally, the improved method provided size distribution and density gradient data. This tool played a pivotal role in the interpretation of experimental results.

It turns out that most of the aforementioned tempering factors are constrained by either process considerations (incorrect residence time and/or extreme changes in gas blower settings will heat the glass unevenly or get it too hot causing breakage or warp) or machine limitations (limited variability in transition speed and quench air flow). Further complicating experimental design was the fact that the furnace was not owned by SAGE and therefore SAGE personnel had limited influence on how much variation was introduced. Within the allowable range of process changes, however, several trials were conducted to determine the relationship between defect formation and individual tempering factors. No substantial effects were discernable. The next step should have involved more extreme changes to the process, but as already noted this was not possible. However, it is perhaps significant, that with moderate adjustments to the standard operating conditions, no connections could be established. Substrate glass has been tempered for years with very little variation in the recipe and large changes in the defect density can still be correlated to tempering batch. Alternate investigations were needed to determine if the problem was elsewhere.

As already mentioned, substrate tempering is only one part of the processing that all substrates undergo prior to thin film deposition. Because this is typically a 'batch' operation, it is difficult to separate contributions to the defect population from other substrate preparation activities. The first attempt to de-couple tempering from other procedures involved combining two small substrate preparation lots (processed anywhere from days to weeks apart) into one large tempering batch. Defect data from devices made on these substrates was then analyzed to 
determine if there was a statistically significant difference between the dot densities in the two lots. The experiment was repeated several times and no difference was found.

A more direct approach was also employed to look for contributions from each individual step. In each case, processing was modified to alternately exaggerate and minimize the possibility of creating defects. For example, some substrates were covered during the edge seaming operation to minimize the amount of glass chips landing on (and presumably damaging) the surface while others received 10 times the normal amount of edge seaming and were not covered. Once again an analysis of the dot densities revealed no correlation with the processing except for the substrate cleaning. The initial washing experiment showed a significant effect from hand scrubbing the substrate surface (part of the normal cleaning protocol), but when the experiment was then repeated twice in each case it gave inconclusive results.

Other experiments were conducted using smaller, un-tempered substrates to determine a base line level for these non-coloring areas. Previous investigation has shown that defects in the transparent conductive oxide coating (TCO) applied during glass manufacturing can cause some of the dots. The extent of their contribution was, however, unknown. The dots on smaller substrates were individually inspected under a microscope and characterized by root cause. Those caused by microscopic surface damage (typically found after tempering) accounted for only a few percent, further reinforcing the tempering connection. Dot densities in general were very low, which is consistent with historical data for devices made on un-tempered substrates. It should be noted that un-tempered substrates undergo all the other preparation operations (except for the screen-printing of bus bar material, which is unlikely to contribute to these defects).

An important part of the investigation into how tempering contributes to the evolution of defects was an improved substrate tracking system. Tempering order (identifying which substrates went through the oven first, second, third, etc.) and oven position (typically, six substrates were placed in the furnace at one time) were recorded during each session. Occasionally, a substrate would break during the quench phase and devices made on substrates adjacent to it would usually have high numbers of large dots. Close inspection revealed substrate damage at the center of these dots (presumably caused by debris from the broken glass).

Broken glass is an inevitable part of virtually all tempering operations. The quench areas typically contain thousands of glass particles ranging from 'chunks' to microscopic dust. It is quite likely that airborne glass dust becomes entrained in the high velocity quench air and impacts on the substrate surface. In the tempering plant used for these early experiments, the quench air was not filtered. Any particulates in the incoming quench air or in the ductwork would inevitably be hurled against the substrate at high velocity, thereby probably leading to damage which it is known can result in a non-coloring area. 
A calculation ${ }^{\S}$ shows that a 5 micron particle traveling at 44 mph can create a 0.5 micron deep crack in a piece of float glass (Figure 17). The calculation is based on annealed float glass at room temperature. The presence of the TCO coating and the significant changes in the properties of glass at tempering temperatures would no doubt radically alter this calculation. However, velocities of the quench air during the tempering of $3.2 \mathrm{~mm}$ glass approach $300 \mathrm{mph}$ and it seems likely that under these conditions, even very small particles can breach the sodium diffusion barrier deposited by the manufacturer. A new mechanism is now suggested to explain the connection between tempering and non-coloring device defects. When ambient particulates in the quench air reach a critical size and/or density, substrates are littered with potential defect sites. This may account for the seemingly random nature of the tempering effect.

With the information relating the observation of surface damage to the occurrence of non-

Velocity vs. particle size to initiate a 0.5 micron deep crack in annealed float glass at room temperature

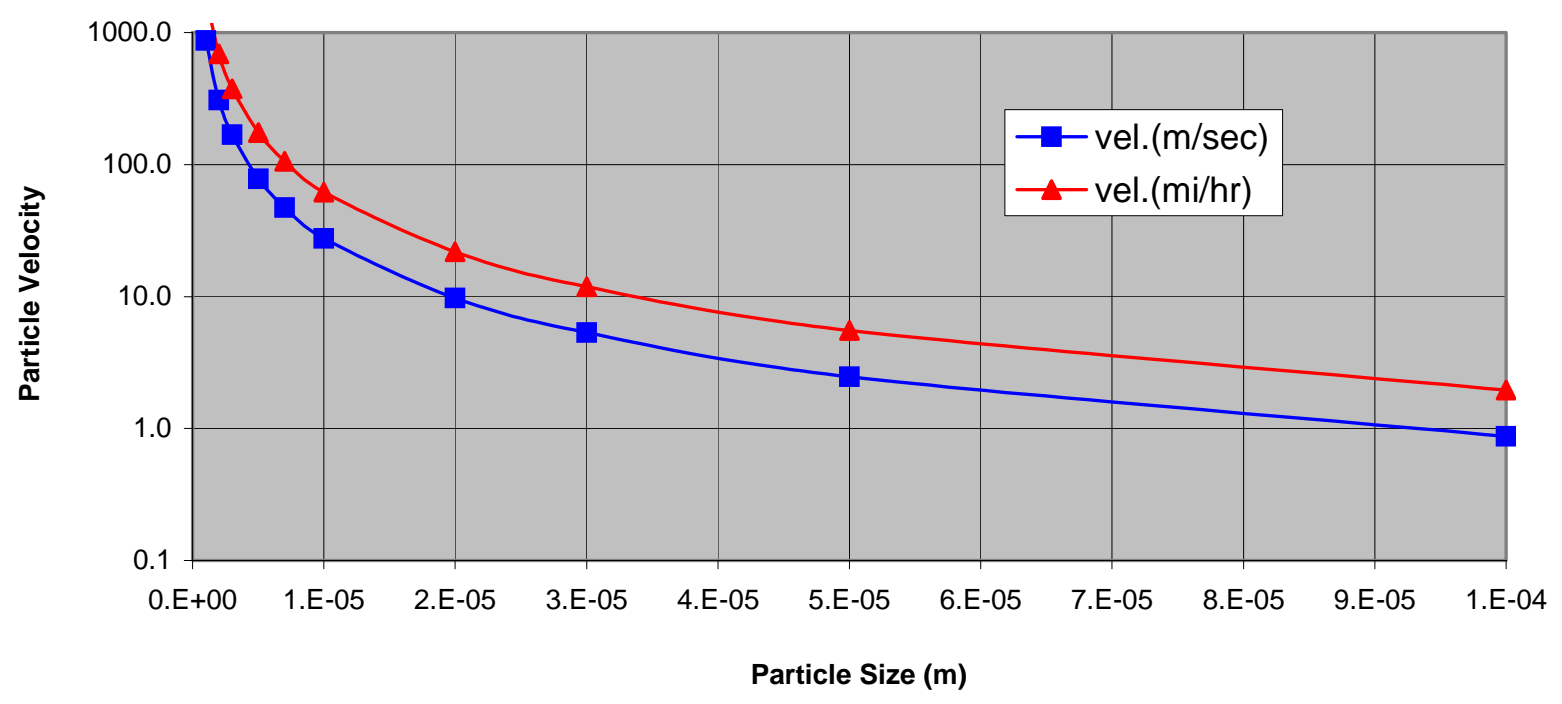

Figure 17 - Literature data showing velocity vs. particle size for an impact causing a $0.5 m i c r o n$ deep crack in a glass surface.

coloring areas, the tempering furnace at SAGE’s new Production Facility was designed with an aim of minimizing the potential for introducing contamination into the quench air stream.

Since installing the tempering furnace in the Production Facility at SAGE, the level of noncoloring areas has been dramatically reduced. This can be attributed to the care with which the system has been designed and installed. In most tempering systems, the quench air is drawn from outside of the building, as the volume required would seriously affect the building air handling systems. In most cases, this introduces serious contamination, and would therefore cause a significant problem for EC device production. However, as an example of the attention paid to this matter by SAGE, the entire outside wall of the tempering facility is made up of $5 \mu$

\footnotetext{
$\S$ Based on the paper "Strength Degradation of Glass Impacted with Sharp Particles: I, Annealed Surfaces",
} Wiederhorn and Lawn, J. Am. Ceram. Soc. Vol. 62, No. 1-2, Jan-Feb 79. Pp 66-70 
filter bags which minimize the amount of contamination coming into the building to be used as the quench air.

\section{III.2.1 Develop Method to Generate Defects}

The objective of this task was to reproducibly generate defects in the sodium barrier layer applied to the substrate glass of test substrates. The test substrates would then allow process modifications to be evaluated according to their efficacy at suppressing the subsequent development of the defects into non-coloring dots. This task was accomplished to complete satisfaction, ahead of schedule, in late 2003. Spark erosion and several mechanical and abrasive techniques were evaluated, but were found to be irreproducible or impractical. Ultimately, it was found that an electrostatic discharge (ESD) generator provided a reproducible and convenient means for generating defects that penetrated the sodium ion barrier layer applied to the glass substrate. In order to generate regular arrays of defects on diagnostic test substrates, an ESD electrode was mounted on a lead screw translation stage. Figure 18 illustrates the result.

Briefly, contaminant ions bloom into the EC layer from the soda lime glass through damage sites in the FTO layer. The size of the non-coloring dots corresponds to the size of the contaminant bloom. Defects in the FTO layer can be generated artificially using an electrostatic discharge so that the effects of processing conditions on the size of the contaminant bloom can be investigated more readily.

In order to facilitate defect generation, an electrostatic discharge (ESD) electrode was mounted on a motorized, lead screw driven translation stage. With a constant discharge rate of $2 \mathrm{~Hz}$, a regular row of ESD damage sites could be scribed reproducibly onto FTO coated glass substrates. The discharge gap distance could be varied precisely, in order to obtain precise control over the discharge potential and relative size of the damage sites. Both the digital characterization and ESD damage techniques facilitated process experiments.

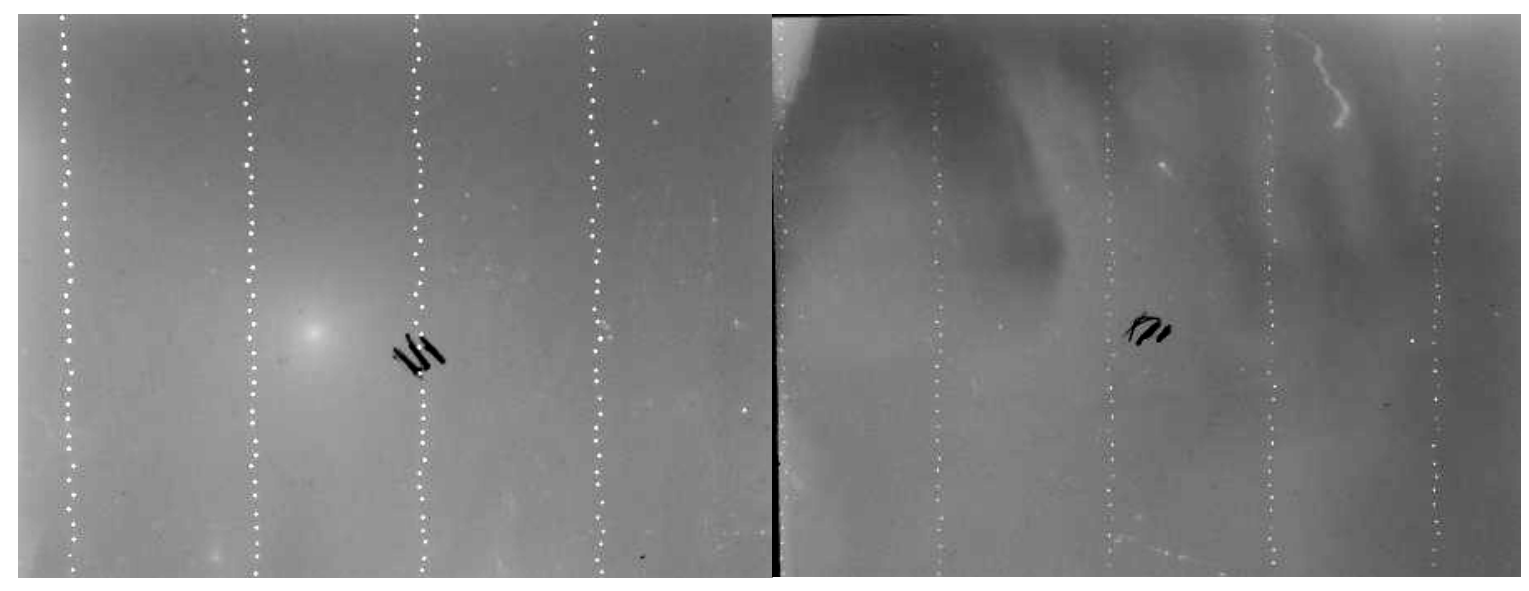

Figure 18 - (Left) An array of non-coloring dots on an EC control device. Defects were created in the sodium barrier layer on the glass substrate by ESD. The image contrast has been enhanced. Note the prominent non-coloring dots. (Right) An array of dots similar to those in the left hand figure, except that the proprietary EC layer has been leach treated. The image contrast has been enhanced to reveal the rinsing marks. Note that the non-coloring dots have been suppressed. 
In order to rapidly and accurately quantify the effect of process changes on the development of non-coloring dots, a digital 'dot-meter' was also developed. The dot meter consisted of a digital camera mounted on a large, pyramid shaped lens-hood that served to maintain a constant focal length while eliminating stray light. Image analysis software was configured so that defect statistics could be rapidly and conveniently obtained from the image data. The combination of the dot meter and ESD generated defects has provided a valuable tool for evaluating process improvements.

For example, in an attempt to generate larger non-coloring dots, to further explore the effects of processing conditions, dots on demand test coupons were heat treated after EC film deposition, but prior to IC deposition. Surprisingly, this did not increase the diameter of all of the dots on the test coupon uniformly, but broadened the dot size distribution. In fact, the diameter of many of the dots on demand was smaller on the heat-treated test substrates than on the respective, experimental control substrates. This would suggest that time at temperature after EC layer deposition is not a determining factor for non-colouring dot size (or the radial extent of sodium diffusion). It has also led us to speculate that sodium diffusion might be accelerated through the sol-gel derived ion-conducting layer while in the gel state.

A combination of further experiments revealed that the soda bloom responsible for the largest excursions in dot density is something that develops while the sol-gel derived ion conductor is in the unfired, wet gel state. This is in agreement with published values for the diffusion coefficient of sodium ions in water (similar to the diffusion coefficient for a wet gel film) and those for the solid-state layers. This realization prompted an analysis of different approaches for eliminating the defect, resulting in the following list:

1. Development of a sputter deposited IC layer to eliminate the wet gel state;

2. Reformulation of the sol-gel coating solution to obtain an anhydrous gel that minimises sodium ion diffusion; and

3. EC layer leach treatment.

Other options, all ranking lower on the cost/benefit scale, include substrate screening (for FTO defects), substrate cleaning (to minimize sodium sources), optimization of substrate preparation and tempering, and alternatives to the current substrate supplier.

Ongoing quality improvement projects are continuing on several of these topics, particularly the sputtered IC layer, as well as the tempering process optimization, but the overall problems due to the non-coloring dot defect appear to be at a level which will allow us to ship saleable product from the Production Facility. This effort can therefore be declared successful.

\section{III.3 Evaluate lon Exchange Layer}

The objectives of this task were to evaluate the feasibility of a protective, temporary salt or glass layer, to be applied to the substrate surface prior to tempering, by spray coating or spin coating and then stripped off after tempering. The objective was achieved in part, and it was found that the concept is indeed feasible. Lithium and potassium bearing materials could be dip cast onto the substrate, survive tempering, and then be stripped off with a caustic cleaner, with little or no 
detectable degradation of the substrate. However, the efficacy at protecting the substrate from damage and suppressing non-coloring dots was not evaluated.

It is thought that at temperatures encountered in the tempering process, lithium and/or potassium ions from the protective, strippable salt/glass layer will readily exchange with the offending sodium ions in the vicinity of defects in the sodium barrier layer. It is also thought that the strippable layer will provide a mechanical cushion to particle impacts in the quench section of the tempering process, where the substrate has very little fracture toughness.

It was found that although this offered a potential method for reduction in the amount of sodium left on the substrate after tempering, and therefore available to contaminate the films, the work on the reduction in the number of damage sites due to tempering issues has been reduced to such a degree, that it was thought that this did not offer a big enough return for the amount of time which would be required for it to be developed fully.

\section{III.5 Post EC Layer Chemical Treatment}

The objective of this task was to suppress the non-coloring dot defect, without incurring side effects, by selectively leaching sodium ions out of the proprietary EC layer. Non-coloring dots have been successfully suppressed by subjecting the proprietary EC layer to anodic leach treatment. However, side effects associated with the leach treatment and subsequent rinsing and drying, while substantially minimized, remain to be eliminated.

It should be noted that the digital dot meter and test substrates with ESD generated defects, described previously, were used to great advantage. These tools were used to optimize a process for selectively leaching sodium ions out of the proprietary EC layer, in a series of statistically designed process experiments, or DOE experiments, in order to suppress the non-coloring dot defect.

Essentially, test substrates coated with proprietary EC material were immersed in experimental leach baths, rinsed, drained dry, and then returned to the coating process to obtain complete devices. Several key, proprietary parameters were identified. The leach treatment was iteratively optimized according to the following criteria: maximum suppression of non-coloring dots, minimum development of visible side effects, and shortest possible leach time. Numerical scales and weighting factors were assigned to these criteria to obtain a desirability function. An example of how the desirability function responded to two of the key leach treatment parameters is depicted in Figure 19. Clearly, a maximum desirability was obtained for moderately low $\mathrm{pH}$ and the shortest leach time. 
DESIGN-EXPERT PIot

Desirability

$\mathrm{X}=\mathrm{A}: \mathrm{pH}$

$Y=B:$ time

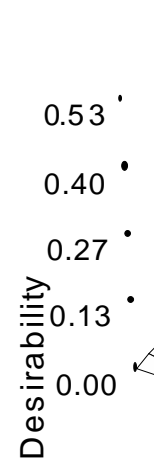

$1.00^{\circ}$
B: time

Figure 19 - An example response surface for a desirability function for leach treatment conditions. Process desirability is plotted on $z$ axis as a function of leach bath $\mathrm{pH}$ and leach time.

A comparison of the photographs in Figure 20 illustrates the effectiveness of optimal leach treatment, on the macroscopic scale. The figures illustrate the difference between untreated (Figure 18 left) and leach treated (Figure 20 right) EC layers that have been incorporated into EC devices. Clearly, leach treating the EC layer suppressed the development on noncoloring dots, which are only faintly visible in the contrast enhanced image on the right. However, swirl marks or water marks are visible. These have been
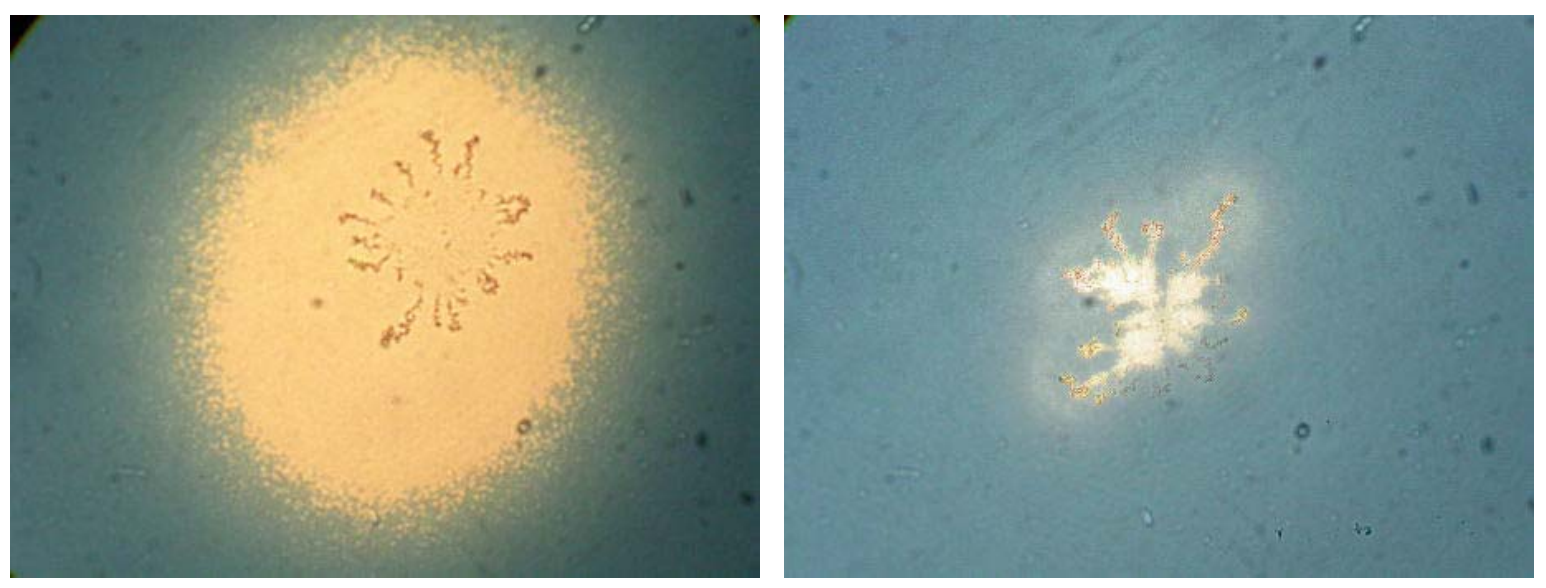

Figure 20 - (Left) Photomicrograph of a non-coloring dot that developed from an intentionally generated defect (by ESD) in the sodium barrier layer, and (right) the effect of EC layer leach treatment on the development of a noncoloring dot. 
traced to the rinsing and drain-drying steps for the leach treatment, which have yet to be perfected.

A comparison of the photographs in Figure 20 illustrates the effectiveness of optimal leach treatment on the microscopic scale. The figures illustrate the difference between untreated (Figure 18 left) and leach treated (Figure 18 right) EC layers that have been incorporated into EC devices, now at $150 \times$ magnification, where the width of each image corresponds to a distance of $0.5 \mathrm{~mm}$ on the EC device.

Clearly, the non-coloring dot extends well beyond the damage site for the untreated sample. By contrast, the non-coloring area has been confined to the damage site for the leach treated sample. This effect corresponds to the selective removal of a sodium ion stain, or soda bloom.

\section{Summary of efforts to reduce occurrence of light spot defect}

At the beginning of the project, the origin and formation mechanism for the light spot defect were unknown. The defects were also endemic to the existing process, came and went sporadically and threatened to dramatically reduce yields. At the time of writing, the formation mechanism can be said to be relatively well understood. This is as a result of a combination of high quality analytical research, along with a largely empirical DOE approach. As a result of

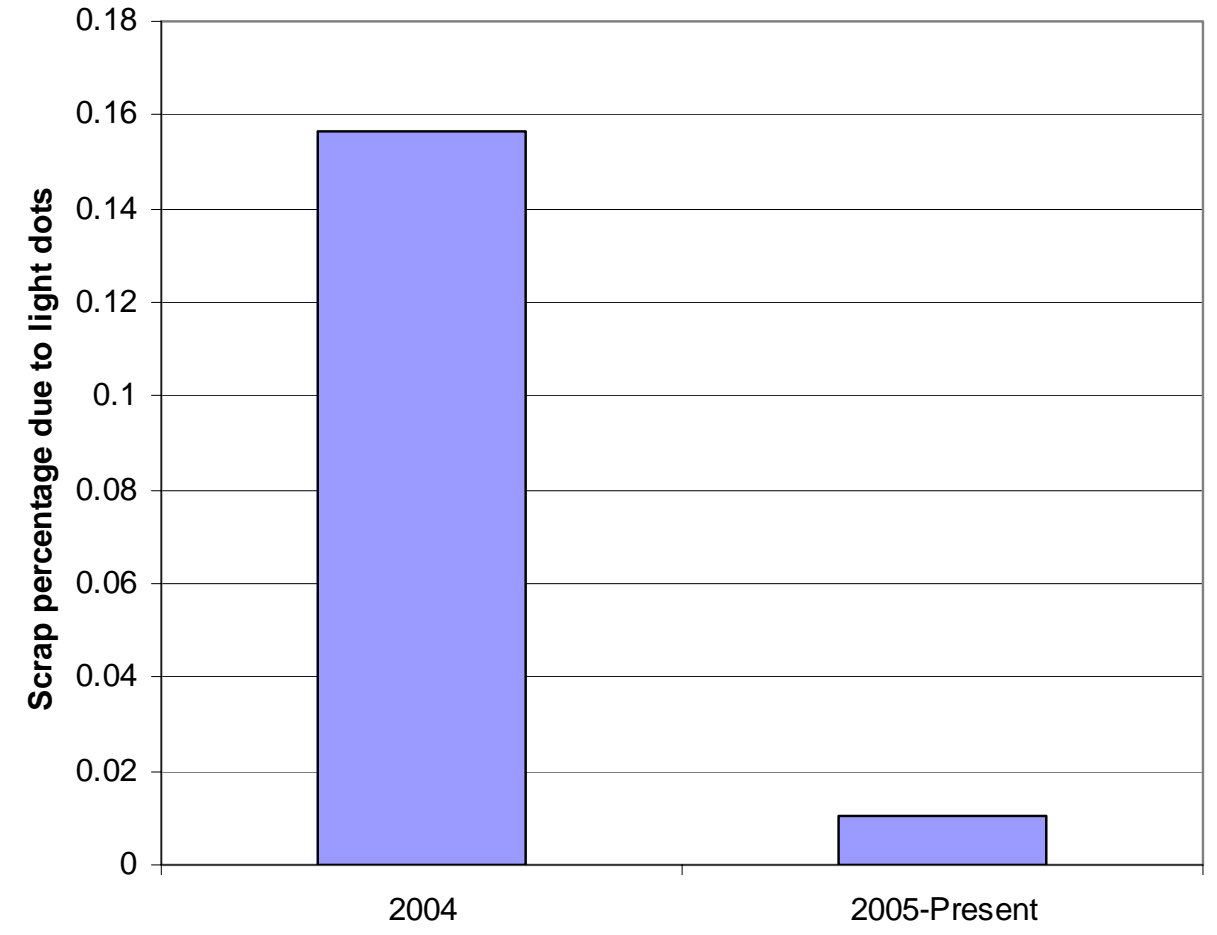

Figure 21 - Devices rejected for the white dot defect prior to the project and after implementation of the recommendations of this project. this understanding, steps were taken to design the new tempering furnace to minimize the potential for substrate damage, by maintaining the highest possible level of cleanliness in that process, and particularly in the quench air.

Also, the number of devices rejected for the light spot defect has been dramatically reduced as a result of this work. To illustrate this, of 595 devices labeled as production (i.e. excluding $R \& D$

devices) in 2004, 93 were scrapped because of excessive numbers of white dots. This represents approximately 15\%. Since moving into the new Production facility, of 5349 devices labeled as Production, only 55 have been rejected for the light dot defect, or around 1\%. Figure 21 
illustrates this. This represents a dramatic improvement in yield, and can therefore be said to be a major success for this project.

\section{III.7 Develop techniques for repairing or excising electrical short defects}

The following sections describe the work carried out on the second type of defect identified as being a major cause of potential yield loss - the electrical short or just 'short'.

A short is a breach across the device between the top and bottom electrical conductors. This breach allows electronic current to flow through the circuit at that point, and prevents a potential difference from being established. In an EC device, the build-up of charge responsible for the coloration change is completely dependent on the voltage established between the upper and lower transparent conductors. An absence of this voltage will prevent coloration from occurring there. Due to the finite resistance of the transparent electronic conductors, current flowing towards the short will cause voltage to be dropped, thereby gradually establishing the required potential difference. In this way, the effect of the short will appear to gradually fade away as the distance away from the defect is increased.

It had been found that it was sometimes possible to eliminate shorts by physically scribing the defect out, using for example a sharp point, but this often resulted in a more severe short, and it was therefore evident that this was not a viable way to remove shorts in a Production environment.

\section{III.7.1 X-Y Stage and Algorithm Development for Elimination of Shorts}

The originally proposed objective was to design and build a stepper motor driven $x-y$ stage and to develop an algorithm to locate the centers of electrical short defects. This would be used in conjunction with micro spark erosion for excising electrical short defects. An $x-y$ stage was built with a short finding algorithm, but it was found only to work on large well-defined shorts. The $x-y$ stage was adequate at the time it was built, but as throughput increased and device specifications tightened, the use of the $x-y$ stage took too much production time and was not as good as the human eye at detecting all types of shorts. However the micro spark erosion technique of excising shorts was found to be a very useful tool with manual rework and was developed further.

Using a new electronic system designed, in part, to help optimize the re-work process, a DOE experiment was devised to explore a variety of electrical and timing parameters. It proved possible, using this system, to re-work defects while leaving consistent non-coloring spots of various sizes $(100-400 \mu \mathrm{m})$, although the smaller ones were associated with a greater probability of failure (either leakiness or failure to adequately destroy the defect.)

This technique has been developed to the point where it is standard for reworking shorts at the monolithic test stage. This is done to enable devices to color as fully as they can, in order to make a judgment on the overall characteristics, in order to determine whether further cost and effort should be expended in fabricating the device into an IGU. Should the properties be such that this is warranted, the device is fabricated, and subjected to a room temperature accelerated cycling procedure (also sometimes referred to as a 'burn-in' process), whereafter the devices are tested again, and shorts reworked using a new proprietary system discussed below. The main reason for developing the new system is to allow for rework within the IGU where the microspark discharge technique is not possible. 
Development of the new system is discussed in the following section.

A new motion control system to be used for removal of shorts from SAGE devices has been designed and constructed. It became operational at the end of August, 2004. Its range of motion is 46 ” $\times 66$ ”, more than enough to cover the maximum anticipated device size during the next stage of SAGE's development. The system consists of three linear translation units mounted in an " $\mathrm{H}$ " configuration, a sturdy system support table, a machine guard safety enclosure, and an optical breadboard mounting surface for the optics. The cross-bar carries focusing optics, the auto-focus system, and a narrow angle viewing camera. A wide-angle camera is mounted high in the safety enclosure, giving a view of the full device being processed while eliminating the possibility of a technician being exposed to any potential hazard. Power supplies and control and safety monitoring systems reside in a rack enclosure mounted to the support system frame. A control computer sits on a positionable stand, located for the convenience of the technician operating the system.

The " $\mathrm{H}$ " configuration of linear translators was chosen over the $x$ - $y$ table arrangement because of its improved space utilization and lower mass, allowing higher acceleration. With the linear translator combination, each axis needs to be only a bit longer than its travel length. For example, the long translators in this system have a length of $2100 \mathrm{~mm}$ for a travel distance of $1600 \mathrm{~mm}$. The travel distance needs to be only slightly larger than the work-piece. In the case of an $x-y$ stage or table, the system requires a space of at least double the work-piece size just to accommodate the motion. Additional space is still required for support structure. The attendant moving mass results in lower acceleration and slower processing times.

The particular stages chosen offer good performance. Drive is by servo-motors via a cog-belt with a semi-cylindrical land tooth configuration. The moving platform is supported and guided on precision ground steel rods. The basic structure of the unit is an aluminum extrusion. Units can be mounted either horizontally or on edge. The SAGE system utilizes both mountings to maximize rigidity over the longest unsupported lengths. A pair of $2100 \mathrm{~mm} y$-axis stages, driven by a common servo-motor, support a $1600 \mathrm{~mm} x$-axis cross stage. The $y$-axis stages are mounted flat, and supported by a series of feet along their length. This configuration makes it easier to mount the cross stage and position the unit on the support table. Shims under the feet will ensure planarity. The $x$-axis cross stage is mounted on edge, as has an unsupported span of about $1200 \mathrm{~mm}$, and the edge configuration has about $1 / 9^{\text {th }}$ the deflection for a given load than that realized with the flat mounting configuration. This also puts the $x$-axis stage in a vertical orientation, allowing a simpler mounting scheme for the optics head.

The decision to use this particular unit was made following tests run at SAGE on a smaller version of the " $\mathrm{H}$ " stage. Positioning resolution and repeatability were measured using a microscope and digital camera attached to the stage, while the substrate remained fixed. Moves of $10 \mu$ were possible, while moves of $5 \mu$ or less could not be made. The average repeatability $(1 \sigma)$ of a simultaneous $10 \mathrm{~cm} x$ - and $10 \mathrm{~cm} y$ - move was $11 \mu$. Backlash was about $12 \mu$. Estimated resolution required to remove point shorts while maintaining a non-visible spot size of $100 \mu$ or less is about $35 \mu$, so this is more than enough resolution for the task. The belt drive is expected to be less durable than ballscrew or linear motor drives, but a more aggressive maintenance schedule, especially frequent belt replacement, is expected to compensate.

The support structure is a framework of 2" wide by 6" deep rectangular steel tubes, for minimum deflection. 4" square steel tubing is being used for legs. Spacings are designed to accommodate 
the stage structure, the optics breadboard, and the backlight for illuminating defects in colored windows. The large section tubing, all 0.25 " wall thickness, provides the stiffness necessary for accurate stage alignment in both static and dynamic loading situations. The largest moving stage unit weighs about 65 pounds, and can be moved at up to 4 meters per second, so dynamic loading was an important issue.

Vision for the operation is provided by two cameras inside the enclosure. A wide-angle camera, mounted high in the enclosure, provides a view of the entire substrate. A narrow angle camera, mounted on the translation stage, provides a view of the process area through the primary lens. These views enable manual operation of the motion system at first, and facilitate the eventual conversion of processing to machine vision control.

The plan evolved for the rework system is to use the technician's trained eye to guide the laser head to the rework location, using the monitors and internal cameras to provide a view of the work-piece, and mouse and joystick to manipulate the motion system. This will migrate to optical recognition software as soon as possible. Light spot defects are now routinely characterized at SAGE by optical recognition, so it should not be prohibitively difficult to identify some percentage of defects in this way. The challenge then will be to enlarge that percentage. The defect characterization software also outputs defect locations, which can be developed into guidance for the rework system. This work has been done with the existing manual rework stations, and some work will be needed to migrate this to the new rework station now under construction.

\section{III.7.2 Micro Spark Erosion Rework}

The main issues with spark erosion rework are the size of the ablated area, which leads to a nonelectrochromic spot, and hence a light dot, and whether the reworked area becomes leaky during product screening. Reworked areas have missing films or no films at all and hence do not color, and then appear as a non-coloring dot, ideally the size should be equal to or less than 300 microns. Also, the reworked area can become leaky after the initial rework attempt and may need to be reworked again. Alternatively, the area can become leaky in the future and in essence become another short that cannot be electronically reworked because the films are now inside an IGU.

Initial testing was done with an in-house designed and built spark generator, but more recent testing was done with an off the shelf electric pulse generator. The adjustable parameters for the pulse generator are voltage, frequency, pulse width, and number of pulses. An initial feasibility test was done to achieve a setting where the initial rework was not leaky. Once a satisfactory setting was found, a range finding DOE showed that the important variables were voltage and number of pulses.

A simple matrix was set up using four small devices measuring rework size and number of leaky reworked areas. Twenty spots were reworked on each device.

It appears that with these parameters leaky reworked areas are not a concern and the size range looks acceptable.

A pair of devices was reworked using a setting of $100 \mathrm{~V}$ and 400 pulses (the highest setting was used initially because the cleared area size range is the same as what is observed with the current system). Neither of the two devices had any leaky reworked areas. Future testing will include 
reworking more devices with the pulse generator to build up a statistical history and lowering the voltage and number of pulses to get the smallest reasonable cleared area possible.

\section{III.8 Develop Efficient Burn-in Test}

\section{III.8.1 Determine reliability of current manufacturing process}

Over the two years following the project commencement a burn-in process was developed, which involved cycling from clear to dark at room temperature and then sometimes at high temperature, ensuring that a total of at least 700 cycles are achieved prior to shipping. This screen was optimized to bring out latent point defects (shorts) for re-work prior to shipping as well as to accelerate other failure modes, such as generation of non-uniformities. The effectiveness of this screen was evaluated by studying the limited number of field failures as well as the distribution of short production as a function of the number of cycles. From this work there was not enough evidence to suggest that the burn-in procedure significantly impacted the expected lifetime of the product. More comprehensive testing is required to determine any effects upon lifetime.

An analysis of the frequency of latent short production as a function of cycles at room temperature was carried out, and it is clear that cycling was reasonably effective at bringing out latency as the instantaneous failure rate falls with increasing numbers of cycles. However, at the end of the standard 700 cycle burn-in test it is clear that there is still a finite probability of additional latent shorts occurring.

\section{III.8.3 Design accelerated test experiments}

Given the nature of this measurement, it was concluded that the present burn-in test was adequate for initial screening of devices prior to shipping. Work continues in an effort to more accurately estimate the lifetime of devices have passed through the test.

\section{III.9.2 Use conductive adhesive bus bars}

Our investigations here were mainly focused on the logistics of handling the adhesive material. It was found that the difficulties of handling the material proved to be substantial, and the efforts required to ensure dispensing equipment that would not require continual cleaning, etc. would more than offset the benefits of such a system.

\section{III.9.2.2 Evaluate embedding copper wire in adhesive}

Due to the issues with handling the conductive adhesive discussed above, this issue was given little attention. However, it was felt that one additional major hurdle would have to be addressed - given the successful implementation of the adhesive technology itself - in that the wire would need to be held in place while the adhesive cured. This is not a trivial matter for mass production, and as a result this approach was not investigated further.

\section{III.9.9.4 Solder attachment of copper bus bars}

A number of approaches to the ultrasonic application of bus bars to electrochromic devices were investigated. 
An initial search of vendors, with a view to finding a vendor or vendors with a well-developed technology for application of metallic bus-bars to a coating surface was carried out. This turned up vendors of ultrasonic soldering equipment and ultrasonic welding, and micro-welding. These efforts were unsuccessful in turning up an existing solution or even samples that looked promising.

Several indium-based solder compositions were investigated, $97 \% \mathrm{In} / 3 \% \mathrm{Ag}$, and a series of solders of the Cerasolzer brand with melting points of $143^{\circ} \mathrm{C}, 286^{\circ} \mathrm{C}$, and $223^{\circ} \mathrm{C}$. In general it was found that the best adhesion to the coatings was with the $97 \% \mathrm{In} / 3 \% \mathrm{Ag}$ solder. The Cerasolzer solders generally increased in hardness as the melting temperature increased, but it was generally found to be more difficult to get a consistent bond to the coating surface.

Ultrasonic solder application of copper ribbon with indium-based solder pre-applied to one side of the ribbon was attempted. An apparatus was made which enabled the operation to be performed with a reasonable degree of control. Tension of the ribbon, force of the ultrasonic soldering head, rate of travel, and soldering temperature and ultrasonic energy level could all be controlled. It was found that the copper ribbon needed to be first coated with 50 microns of Sn or something similar to avoid inter-diffusion of indium and the formation of a brittle intermetallic.

However, the adhesion of the solder to the coating surface was inconsistent, occasionally pulling the coating apart and occasionally pulling easily off of the surface. Often these extremes existed within centimeters of one another on a piece of ribbon. Surface cleanliness, temperature of the glass surface, and the effectiveness of ultrasonic energy transfer were felt to be variables here which might be brought under control with more investigation.

There was a significant problem with splatter of the molten solder ultrasonically vibrated between the ribbon and the substrate. This resulted in droplets of solder material being deposited on the substrate surface where they presented at best a cosmetic problem, and at worst a potential short as the indium diffused into the coating over time. This problem appeared to be quite serious, and it was evident that it would be a considerable effort to find ways to contain it. Various collection techniques were considered but not investigated.

Next, ultrasonic application of solder directly to the coating, and solder attachment of pre-coated ribbon to that was tried. It was found that good, repeatable adhesion of the solder directly to the coating surface could be achieved. Electrical contact appeared to be satisfactory to both conductive layers. Spatter was less of a problem, but still significant at times. This issue is considered to be a major obstacle which would need to be overcome before this technique could be implemented. There was only intermittent success in getting good adhesion of the ribbon to the pre-applied solder trace. The best results were when the ribbon was pre-coated with solder, and this was melted to the pre-applied solder trace. This method appeared to hold some promise, but needs considerable development to determine the ideal process conditions.

Finally, ultrasonic welding was investigated via two vendors, neither of whom had success in adhering metallic ribbon to the surface of the EC coating using this technique. This approach was therefore abandoned.

To summarize, a number of different approaches to solder attachment of bus-bars to EC devices have been investigated. It was found that these approaches either were not suitable, or would 
require considerable development in order to be usable for this application, and would therefore constitute a much longer term project.

\section{Product Improvement to Increase Acceptance to OEM Customers}

An important factor in increasing market penetration of energy saving EC insulating glass units (IGU's) is ready acceptance by OEM customers who assemble them into window frames and by installers and contractors who glaze buildings. Feedback from customers clearly indicated a need to maximize the vision area of the EC IGU. At the beginning of the project, SageGlass ${ }^{\circledR}$ Electrochromic windows had a large unobstructed vision area capable of being switched from clear to dark and a non-switchable perimeter region. The latter area was clear except for obstructions such as bus bars and the spacer unit. Typically the perimeter region is hidden in the frame. However, the EC IGU technology at that time resulted in an undesirable small band of non-coloring glass around the perimeter of the window when installed in an industry standard frame. It was therefore essential to reduce the width of the perimeter region to less than 0.5 " from the edge of the glass, maximizing the vision area and making sure that non-switching clear glass areas are hidden from view in standard frames. The task involved moving the bus bars closer to the edge of the glass and outside of the desiccated and protected spacer environment. Additionally, the sputter masks for the EC layers had to be redesigned to move the films closer to the edge of the glass.

\section{IV.1 Maximize Vision Area}

The progress made on this important aspect is described in some detail in the following sections: IV1.1 deals with relocating the bus-bars, IV1.2 with the new mask design for maximizing the vision area for the process, IV.1.3 with future intentions for eliminating the need for masking altogether, and IV.2.4 with work done in accommodating the wiring in currently used framing systems.

\section{IV.1.1 Move and Test Bus Bars}

Developments in this area are confidential to SAGE Electrochromics, Inc.

\section{IV.1.2 Redesign Masks}

A repeatable precision mask alignment system was designed so that the films could be located closer to the edge of the glass. The coloring area of the device would thus be maximized, while at the same time reliably ensuring that the top transparent conductor does not short to the bottom electrode. In addition, the thin films could not be allowed to encroach on the primary seal, and risk jeopardizing the seal integrity.

One of the major issues that needed to be addressed as part of the new mask alignment system was the thin film fringing causing film overlap and thus leading to the top transparent conductor shorting to the bottom electrode. It was well known that both thin film layers displayed varying amounts of film fringing at the film edges. Four of the most probable contributors to fringing were hypothesized to be:

1. Thermal expansion coefficient differences
a) Soda-Lime Glass
$9.35 \times 10^{-6} /$ degree $\mathrm{C}$ 

b) 304 stainless steel
$17 \times 10^{-6} /$ degree C
c) 410 stainless steel
$10.5 \times 10^{-6} /$ degree $\mathrm{C}$
d) Invar 36
$4.92 \times 10^{-6} /$ degree C

2. Glass movement during coating cycle

3. Overspray caused by gaps between the glass and the masks

a) Glass bowed

b) Mask insert warped or damaged

4. Shadowing of films at the mask edge due to mask thickness

Figure 22 shows a typical example of thin film fringing on the long edge of a device. The mask separation must be kept so that the top-conducting layer will not overlap the bottom insulator, or shorting will occur. The new mask system design must allow enough distance to accommodate film fringing, be repeatable so that the film edge does not shift considerably, keep the glass from shifting relative to the mask during coating, and compensate for glass to mask gaps caused by glass bow or holder frame defects.

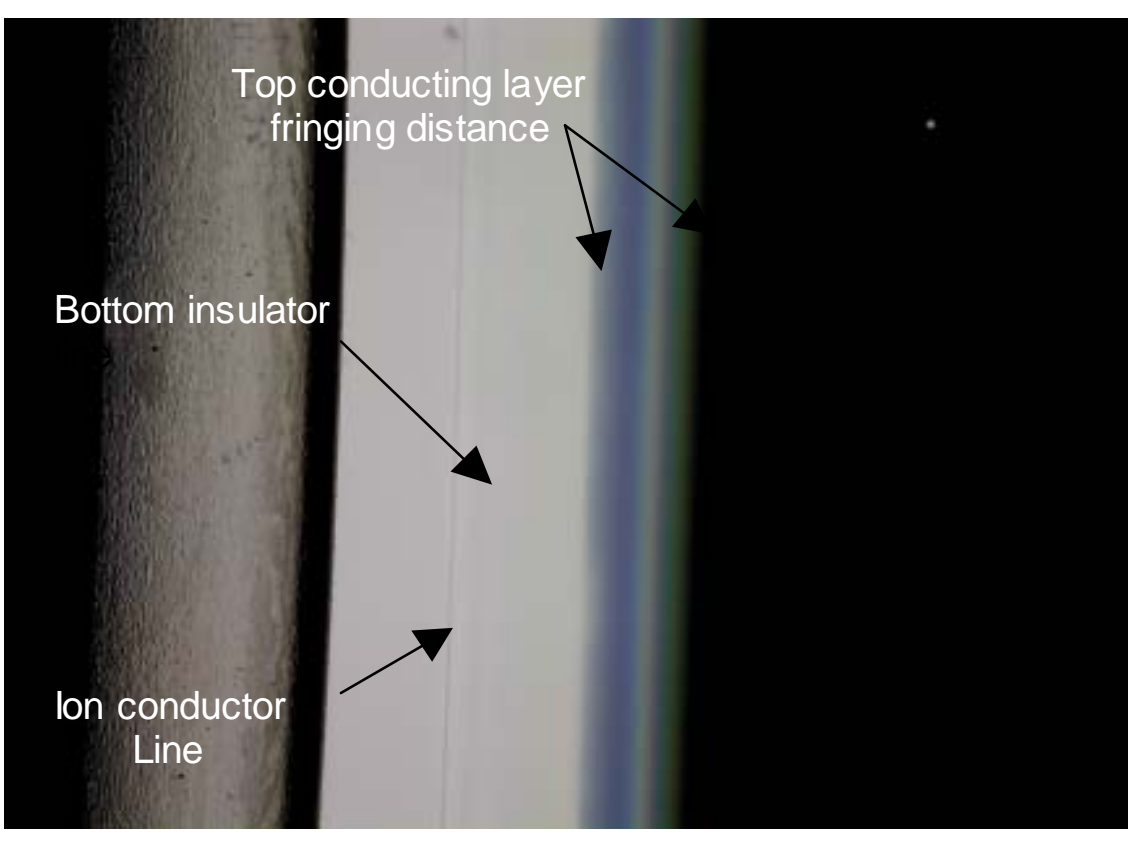

Figure 22 - Typical fringing seen on an EC device.
The chosen alignment system is depicted in Figure 23. The outside frame supports a 0.030 ” thick 410 stainless steel mask that defines the film edges. The 0.030 ” thin masking provides better line definition than one-piece solid masking, since they can be made to conform to a curved surface. Three locator pins in the frame were used to locate each of the masks, and the glass was laid on the mask and located up

against the three pins, which oriented the glass based on two adjacent edges. The same two adjacent edges are also used to align the glass in the operations prior to thin film deposition, from glass cutting onwards.

The bottoms of the 1/8" thick frames had the edges chamfered to reduce shadowing of any deposited films arriving at high angles of incidence. 


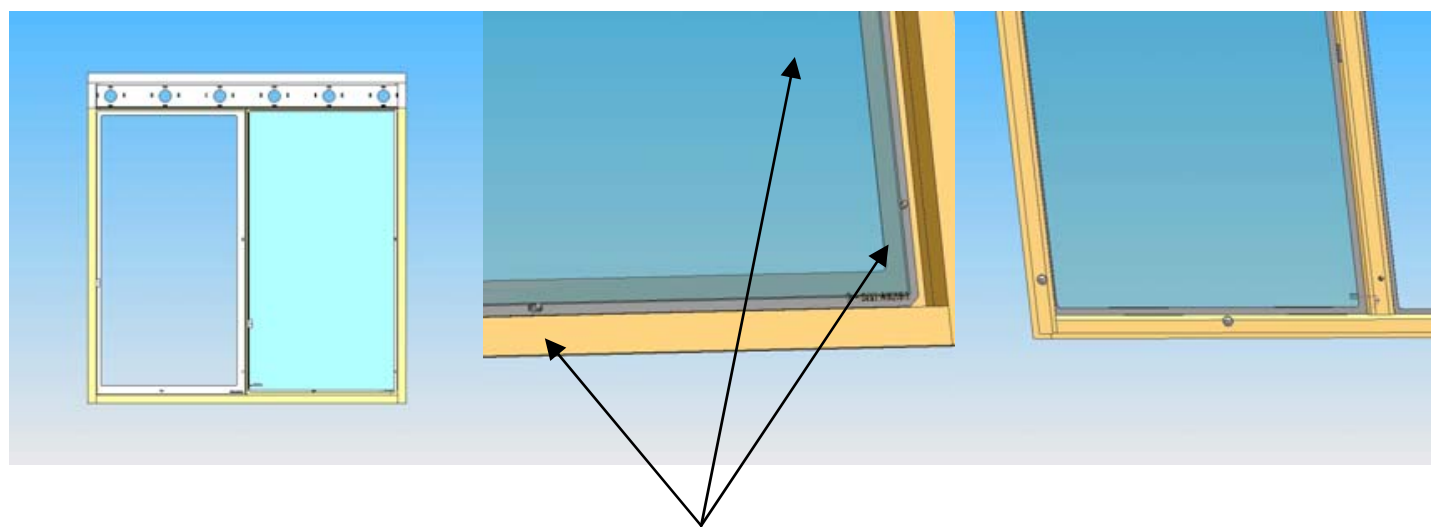

Figure 23 - Diagram showing the location pins used with masks produced during this project. The arrows point to the location pins (the third pin is out of the picture at the top). Note the edges of the masks are champhered to reduce shadowing as much as possible.

Test masks were cut using both laser cutting and water-jet cutting. Although the laser cutting was about half the cost, the slag left behind on the mask was unacceptable due to increased risk of damage to the bottom layer conductor or other films. Water-jet cutting became the approved fabrication method.

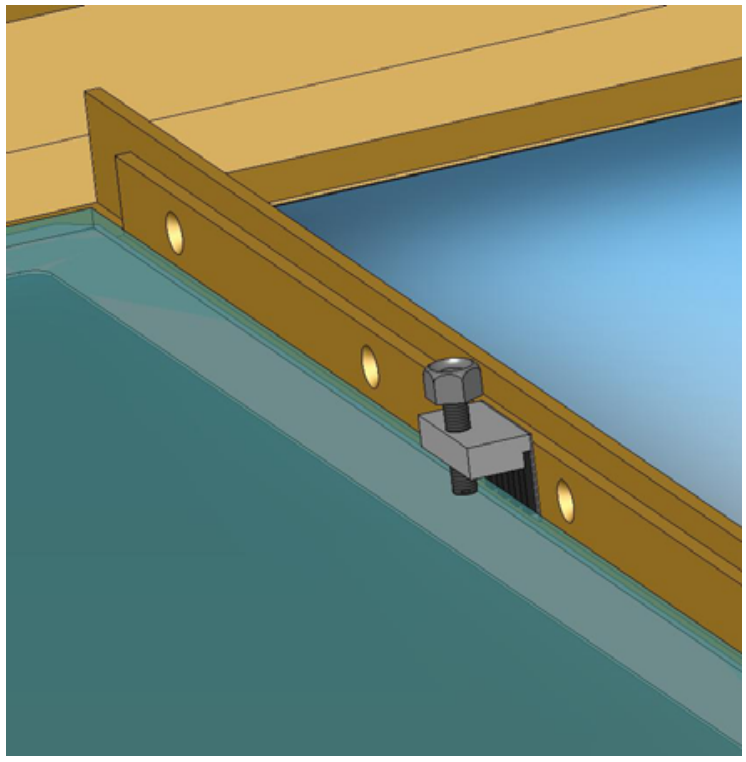

Figure 24 - Detail of a magnet assembly used to hold the mask in close contact with the glass.
However, even the water-jet cut masks were found to cause substrate damage in limited instances. The repeatable position of the damage sites from the same mask, always at the edge of the top conducting film stack, pointed to the root cause, that being scratches from the film mask edges. Both mechanical polishing of the mask edges, and electropolishing of all masks were tested as possible preventative maintenance solutions. It was discovered that mechanical polishing was the best way to ensure that all masks could be used with minimal or no risk of substrate or film damage from the masks. Mechanical polishing of all masks was then incorporated to all newly received masks, as well as newly cleaned masks, prior to releasing them to production. 
Film edge definition using the thin masks is much better on the average than the solid masks, but some areas still tended to show some erratic thin film fringing. Specific areas showing fringing were not consistent from run to run. This pointed to a non-repeatable separation cause, such as glass bow or inconsistent frame warp.

Two approaches were taken to improve film edge definition and reduce fringing due to mask separation from the glass. Figure 24 shows the magnet assembly that was used to draw the mask up to the glass. The magnets were placed by the operators along three sides of the substrate, and adjusted based on separation distance for that particular spot.

Figure 25 shows a spring clip that was placed between the frame and the mask. The weight of the glass compressed the spring clips, placed at set intervals along all 4 sides, to conform the mask to the glass.

Both approaches showed measured improvements in the film edge definition by effectively decreasing the gap between the mask and substrate. The springs under the mask proved to be much less labor intensive, so were thus chosen as the permanent design path.

The finished design moves the top conducting film stack (device coloring area), approximately $11 / 32$ " closer to the glass edge on the long sides, and approximately $1 / 8$ " closer to the glass edge on the short sides of the 17.98” × 34.6” device pane. Film edge positioning variation was verified to be within the tolerances allowed by the bottom insulator and top conductor mask separation via film edge measurements of more than 25 individual units. A quality check has been implemented to verify no films encroach on the primary seal. To date, no instances of noncompliance have been discovered.

\section{IV.1.3 Eliminate Masks}

Laser film definition and large area film removal techniques will need to be developed to achieve the required isolation of electrodes and selectively remove unwanted films in border areas. Work to develop selective laser film ablation was primarily carried out under NSF SBIR contracts. A robust mechanical edge deletion process was developed under this NETL project. We describe elements of both work programs below.

Optical analysis at SAGE and laser evaluations at manufacturers and job shops has shown that a Qswitched frequency doubled 532nm Nd:YAG or $\mathrm{NdYVO}_{4}$ laser is ideal for device edge definition and removal of electrochromic films from spacer bond areas of UGu's. A cost model developed for

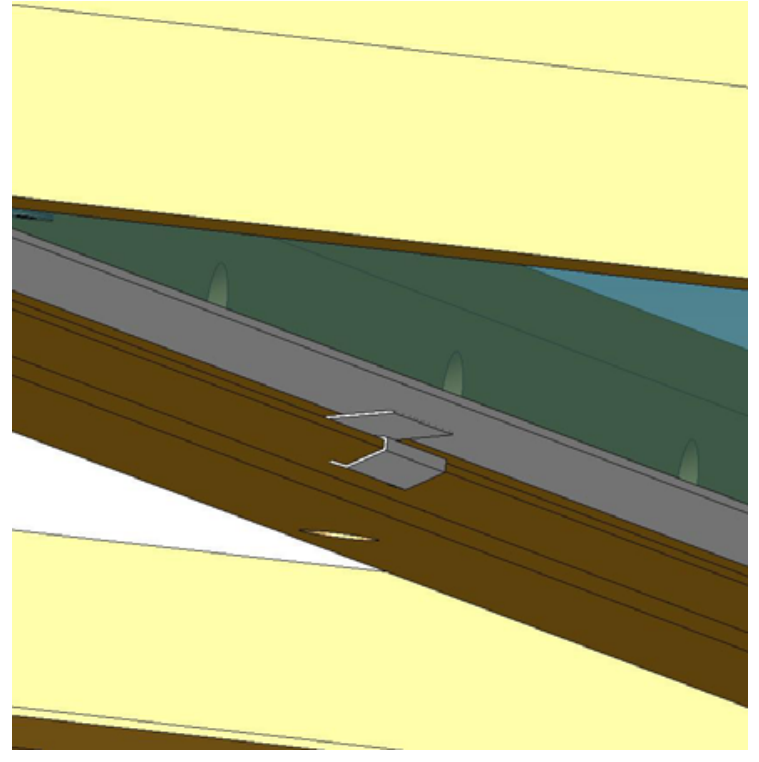

Figure 25 - detail of a spring clip assembly, also used to hold the mask in close contact with the glass. the process shows that speed and throughput are the critical parameters in developing an economically viable process, indicating that a substantially more powerful laser of the same type may be needed for edge deletion.

A SAGE insulated glass unit (IGU) consists of a SAGE monolithic electrochromic device and a plane glass lite, separated by a metal spacer. The spacer is attached with a dual adhesive system, 
a primary bond of PIB as a moisture vapor barrier, and a secondary bond of silicone as a liquid water barrier and mechanical attachment. Tests have repeatedly shown that neither good moisture seals nor good mechanical attachments can be made to sputtered electrochromic films in the edge seal areas.

Additionally, sputtered upper transparent conductor (UTC) films must not end up touching the lower transparent conductor (LTC), or shorting will occur. It is therefore necessary to eliminate deposited films from the edge of the device. This was achieved via shadow masking at the beginning of this project.

Shadow masking has a series of difficulties, sufficient to drive SAGE to look for alternative solutions. In order to produce a crisply defined edge, the thin mask edge must remain in intimate contact with the glass during sputtering. This is discussed in some detail in IV.1.2 Redesign Masks.

The initial SAGE process required one mask for the electrochromic film deposition process, and a separate, smaller, mask for the remaining deposited films, including the UTC. This dimensional difference was to ensure there was no shorting between UTC and LTC, and had the negative effect of increasing the non-coloring area at the edge of the device. This requirement also doubled the number of masks required for a process. Several additional masks for each glass size will be required for production operations to enable the coater to be kept fully loaded for production efficiency, to be pre-mounted with pieces of glass to improve turn-around time, and to allow cleaning without interrupting the production schedule. Not only does this multiplicity of masks become expensive to purchase initially, but storing, inspecting, and maintaining them becomes a costly problem. As the SAGE commercial business develops, the number of different glass sizes is expected to increase rapidly, exacerbating the difficulties.

Laser ablation was considered as a way to eliminate masking problems from the process, and various lasers have been evaluated for ability to remove sputtered films from the electrochromic device without damaging the LTC film. Using a laser with a high ratio of selectivity for the film stack with respect to the lower conductor allows sputtered film removal at flux densities less likely to damage it. We have determined that the maximum selectivity is available at $532 \mathrm{~nm}$, the frequency doubled YAG wavelength. A Q-switched laser produces greater thermal shock in the irradiated film compared to a continuous wave (CW) or long pulse unit. Observations of the debris from several laser patterning tests to date have shown that, under appropriate conditions, significant amounts of the film stack are removed as flakes, rather than vaporized, while preserving the underlying electrical conductivity. This technique allows lower energy pulses to be used to remove the film stack than would be required for film vaporization, again lessening the chances of damaging the underlying conductive film. A challenge with using the laser in this manner is to collect such flakes before they can be re-deposited on the device surface. Air flow control and filtering are expected to address this issue. Some antistatic measures may also be necessary.

Although in principle, it is possible to develop a laser process for removing the unwanted films from the seal area, it is a formidable challenge for the reasons outlined above. It was also found that using abrasion only to clear the seal areas did not work, as it resulted in highly leaky edges, as the mechanical damage resulted in a shorted device. It was therefore decided to adopt a safer approach to the issue. The method selected was a combination of laser edge definition, i.e. using 
the laser to define the edge of the coloring area, and conventional abrasive edge deletion to remove the films from the seal area.

Laser edge deletion was accomplished using the frequency doubled YAG laser as described above. The laser is driven around the perimeter under computer control. Edge deletion of thin films in the seal area (around the perimeter of the device) is carried out using a mechanical abrasion wheel.

Tests on abrasive removal of the films were conducted using a type of abrasion wheel commonly used for "soft low-e" film removal on window glass with sputtered low emissivity films. The wheel was operated at $3250 \mathrm{rpm}$, in the range recommended by the vendor's technical service personnel. Glass was translated under the wheel on a linear stage supported by guide rails, moved by hand. This configuration has the effect of putting pressure on the abrading medium which depends on the spacing of the glass and wheel, and the compliance of the wheel material.

When the compression of the wheel is properly adjusted, by varying the ride height of the wheel, a narrow band is obtained in which the deposited films, including the electrochromic layer, are removed, without a lot of damage to the underlying pyrolytic lower transparent conductor (LTC). On one side of this band, where greater pressure was applied, is a region in which the LTC is completely gone, as determined by electrical resistivity measurement. There are patches and streaks of fused wheel material in this area as well, indications of excess pressure. On the other side of this band, where less pressure was applied, is a region with a sort of smeared variety of films are present, and the resistivity is highly variable.

Wheel abrasion appears to work, but there are several difficulties to be overcome to make it practical. Parallelism of the wheel face and the glass to be processed is critical to achieve uniform results. An apparatus that controls the pressure, rather than the wheel-glass spacing, would be very useful. Excess pressure results in melting of the surface of the thermoplastic Scotchbrite wheel structure, depositing some material on the glass surface, so pressure control is critical. More compliant wheel structures may be useful in this regard. The wheels need to wear slowly to continually uncover fresh abrasive material. Means need to be found to prevent redeposition of wear debris on the device bonding surfaces. Scratching of the underlying LTC needs to be minimized to maintain electrical performance. The scratches come from the grit, rather than from fragments of film being removed. There is no good solution to this problem, except to use the finest grit wheels available.

A second set of abrasion trials used various types of 5" sandpaper disks. An important difference with these types of abrasive media is that the grit is bonded to the surface with thermoset resins, which do not melt and are far less likely to leave deposits fused to the surface being abraded. A variety of grit sizes and compositions were tried, including ungraded calcium oxide, $0.5 \mu \mathrm{m}$ chrome oxide; $3 \mu \mathrm{m}$ and $9 \mu \mathrm{m}$ aluminum oxide, with unstructured, "soft" resin bonding; and $0.5 \mu \mathrm{m}, 5 \mu \mathrm{m}$, and $10 \mu \mathrm{m}$ aluminum oxide in a "Trizact" configuration. Trizact is an abrasive structure in which a hard thermoset resin containing abrasive grit is micro-replicated into arrays of pyramids on the support film. In general these structures are characterized by material removal rates that depend on the size of the pyramids, and surface finishes that depend on the size of the grit. In traditional abrasives, grit size controls both simultaneously. The $5 \mu \mathrm{m}$, and $10 \mu \mathrm{m}$ Trizact disks had rather large pyramids, while the $0.5 \mu \mathrm{m}$ disks had very small pyramids. 
Trials with 5" disks were conducted using a hand tool rotating at 1100 rpm. "Light” pressure was applied by "feel". The heavily structured Trizact disks, with $5 \mu \mathrm{m}$, and $10 \mu \mathrm{m}$ aluminum oxide grit, readily removed the electrochromic film alone, the entire deposited film stack, and the LTC. None of the other compositions removed significant amounts of any of the films, except to polish off the minor asperities inherent in the LTC structure. Using the heavy Trizact disks, it was possible to remove the deposited films without serious LTC removal. This was determined by electrical resistance measurements. Careful control of the abrasion time is required to achieve this. There is much less redeposition of debris than with the thermoplastic Scotchbrite configuration, but it is not zero.

In practice, devices are fabricated so that in a two bus-bar configuration, the bus-bar nearest the edge does not require edge deletion, as there are no films that which need to be removed. In a three bus bar device, the device is processed so that the edge which has the connections made to it is only isolated up to, but not over the bus bars, to maintain the integrity of the bus bars.

Summary: In summary, a two-stage process has been developed using first, an abrasive removal of the moisture permeable films from the edges of the device, thereby allowing standard IGU fabrication techniques to yield units with highly durable seals. Secondly, the electrical isolation is achieved by using a proprietary laser scribing process.

\section{IV.2 Interconnections and Wire routing}

\section{IV.2.1 Select Lead $(\mathrm{Pb})$ free Solder System}

In order to fit with upcoming regulations, it will be necessary for the EC-IGU product to use a lead free solder. This task was aimed at producing a solder joint using such a solder which will give acceptable performance in terms of fabrication, performance, and durability.

We worked with some well known companies specializing in Solder and Brazing techniques to develop a lead-free solder preform and soldering process to fit with our new manufacturing line.

Development work was done using two lead free alloys: Sn96 AG4 (solid wire form), and Sn96.5:AG3.5 (washer shaped preform). Both groups used a Rosin flux to aid soldering. The preforms were soldered at a vendor's location, while the solid wire samples were done at SAGE. Pull tests on the preform samples showed typical bond strengths of around $5 \mathrm{lbs}$ from a 90 degree pull (normal to glass surface); with the exception of one pull measuring almost 15 lbs. The failure modes were adhesive, either wire to solder or solder to the bus bar, with the exception of the $15 \mathrm{lb}$ pull, in which the wire snapped.

Samples hand soldered using the wire roll and a soldering iron, revealed the increased difficulty of soldering with lead free as opposed to lead based solders. Lead free $\mathrm{Sn} / \mathrm{Ag}$ solder requires a higher temperature than the traditional $\mathrm{Sn} / \mathrm{Pb}$ solder. The bus bar is more likely to rapidly leach into the solder at the higher temperatures. So, for non-lead solders, the temperature, soldering time, and solder volume must be carefully controlled. The strongest and most consistent bond was obtained using a tip temperature of $775^{\circ} \mathrm{F}$, tinning the bus bar and the wire separately, and then joining the tinned samples. Care was taken to supply just enough heat while tinning the bus bar to ensure a good bond. Using this procedure gave 5 consecutive pull strengths ranging from 10-14 lbs, in each case the failure mode being cohesive failure at the glass beneath the bus bar. 
We are now completing the purchase of a complete lead free soldering system, with controlled positioning and heating capabilities. Although such a system is much more expensive than a manual solution, it is necessary to ensure repeatability of a strong joint when using the lead free solder.

\section{IV.2.4 Develop wire routing scheme}

There are two distinct applications for IGU type products. The first is in residential markets, consisting basically of skylights and vertical windows. The second major application is in commercial buildings, where large glazed areas are now very common. Each different application poses a series of wire routing challenges. For residential buildings, there are many different window configurations: e.g. double-hung, casement motorized opening, etc. In each case, the issues are: allowing unobtrusive integration into existing framing systems, accommodating the electrical connection, and facilitating ease of installation and replacement.

Preliminary work was carried out with major window and skylight manufacturers to determine the optimum location for the wire, and to enable prototype systems to be constructed. Further work focused on 'Voice of the Customer' surveys, where installers and builders were interviewed to determine their reactions to the product. Extremely useful information was derived regarding location of controls, integration with building electronic control systems, location of connections to the window for ease of installation and removal. Also, ideas regarding making connections to hinged or sliding windows were also forthcoming from these meetings. As a result, further prototype systems were designed.

In the area of commercial glazing, the problems are essentially the same: to enable simple installation of the windows and ensure the wiring is hidden. The framing systems and methods of installation used in commercial buildings are sufficiently different from residential applications to require a different set of solutions. SAGE has contacted two major custom installers to solicit their views on possible integration methodologies. Once again, prototype installations were designed in collaboration with the installers.

One of the issues faced when installing a wired system is coordinating the tradesmen on site, dealing with union issues and/or asking a window installer to do electrical connections (albeit low voltage). We interviewed a number of trades in the industry - glass installers, framing companies who have had experience with PV installation and low voltage contractors, and as a result developed a concept of how the wires will be routed through commercial framing systems to make the installation process run more smoothly. It was concluded that that as much as possible SageGlass ${ }^{\circledR}$ systems be factory built and glazed and transported to the site for installation. This will allow a better control of quality (breakage and electrical connections) as well as removing the issue of who will do the wiring in the frame if site built. In this way the window connections can be tested before leaving the factory, and by providing a simple test box to the installer, the glazing contractor does not have to wait for the low-voltage contractor to come on site before he can confirm that his part of the job is done. The low-voltage contractor and glazing contractor's work can be completed independently (as normal practice). We have also developed approaches for factory-built, site glazed systems and for completely site built systems.

We have developed an integration scheme which can be used for both factory and field glazed systems, which allows easy installation, integration of different trades work packages and pane 
replacement, if necessary. In this solution, we have an IGU with a short ( 6” pigtail), with a factory applied connector.

It is important to have a connector system which has good strain relief, has protection from burrs on holes in the framing system is water resistant and small enough to fit in the edge clearance between the glass edge and metal channel. We identified a slim-line water resistant connector from the automotive industry. We evaluated a number of modifications to the connection system which improve both the water resistance and provide strain relief and protection to the unsheathed wires as they enter the connector. We conducted thermal cycling and humidity tests to determine the water resistance of a number of options and as a result we identified a cost effective strain relief system with improved water resistance and protection from chafing for the pigtail connector system.

Longer frame wire harnesses are then installed- one end of which has the mating connector for the IGU pigtail - running from each of the IGU's through the tubular framing system to the exit point(s) into the interior of the building. The glaziers will run the frame wire harnesses from each window opening to the exit point. They will then glaze the IGU into the window opening, making sure that they connect the IGU to the frame wire harness. This connection occurs either in the glazing pocket itself, but if this area is not dry, then the connection would be made in a dry area just adjacent to the glazing pocket. Any holes required to allow the wires to run would be factory pre-drilled and grommets provided to prevent chafing. Test control boxes could be used to allow the glazing contractors to check their work prior to leaving the site. In the building schedule, normally the electrical system installation is done after the building is enclosed, and therefore after the glazing contractors have left the site. It is important to minimize the installation costs - as labor is the biggest determinant of installation cost, so we need to ensure that the glazing contractor need only visit the site once when installing the windows.

The low voltage contractor will install an electrical box or boxes (depending on the size and geometry of the window or skylight) on the interior of the building in a hidden area (e.g. above a ceiling) near the wire exit point on the frame and feed the loose wires into this box. He will then run additional wires from the location where the control system will be mounted to this junction box. These interior building wires will be connected to the frame wire harnesses in this junction box(es) using standard connection systems.

We have also completed the integration of SageGlass ${ }^{\circledR}$ IGU's into four commercial framing systems with our framing partners. These are being used for training of installers to demonstrate the integration system and the process of installation.

We are continuing to develop integration solutions with additional framing partners.

\section{Value of this project to SAGE Electrochromics, Inc.}

The overall goal with respect to the U.S. Department of Energy (DOE) is to achieve significant national energy savings through maximized penetration of EC windows into existing markets so that the largest cumulative energy reduction can be realized. The speed with which EC windows can be introduced and replace current IGU's (and current glazing) is clearly a strong function of cost. Therefore, the objective for the project was to enable cost-effective manufacture of electrochromic (EC) windows through developments aimed at improving processing efficiencies and throughputs - Tasks I, II, and III. It is important that this be achieved without impacting the fundamental window performance requirements (i.e. optical quality, dynamic range, speed of 
response, etc.). The proposed work also ensured that EC dual pane IGU's could be easily integrated into existing window product lines, including skylights, vertical windows, doors, etc., thereby avoiding significant resistance from the Window Companies to adoption of this new technology. Task IV focused on EC window designs requiring minimal modifications of existing sashes and frames and minimal extra tooling costs.

The project tasks discussed here covered maturation stages 3-5. Tasks started at Stage 3 or 4, and in some cases have been moved beyond Pilot line stage to full-scale manufacturing. Success criteria were laid out at the beginning of the project, and in general can be said to have been achieved almost completely.

Success Criterion 1 required that the materials and processing technologies starting in Stage 3 must pass the preliminary physical characterization to determine whether they can be used for fabrication into prototypes in Stage 4. In Stage 4, process optimization was carried out through statistically designed experiments to improve device quality. Because significant quality improvements have been achieved, the activity was allowed to pass through to Stage 5 . With the exception of the plasma-assisted deposition of the EC layer, this has all been achieved. We can therefore conclude that Criterion 1 has been achieved.

Success Criterion 2 concerned the assembly processes for high quality IGU's, development of hardware for defect repair, and determination of accelerated conditions for rapid product screening. Each of these tasks has been achieved, leading to implementation into full-scale manufacturing.

The third success criterion involved key product improvements for original equipment manufacture (OEM) acceptability. The activities involved several successful engineering changes to production tools, and so designs have been implemented into full-scale manufacturing, clearly fulfilling the criterion.

It is clear for the previous discussion that significant progress has been made in each of these areas. The project as a whole should therefore be considered to be a success, and the benefit to SAGE can be considered to be a major contribution to the anticipated success of the company. 


\section{Conclusion}

The aim of this project was to investigate possible improvements to the SageGlass ${ }^{\circledR}$ EC glazing products to facilitate both process and fabrication improvements resulting in lower overall costs. The project was split into four major areas dealing with improvements to the electrochromic layer, the capping layer, defect elimination and general product improvements.

Significant advancements have been made in each of the four areas. These can be summarized as follows:

- Plasma assisted deposition for the electrochromic layer was pursued, and several improvements made to the technology for producing a plasma beam were made. Functional EC devices were produced using the new technology, but there are still questions to be answered regarding the intrinsic properties of the electrochromic films produced by this method.

- The capping layer work was successfully implemented into the existing SageGlass ${ }^{\circledR}$ product, thereby providing a higher level of transparency and somewhat lower reflectivity than the 'standard' product.

- Defect elimination is an ongoing effort, but this project spurred some major defect reduction programs, which led to significant improvements in yield, with all the implicit benefits which they afford. In particular, major advances were made in the development of a new bus bar application process aimed at reducing the numbers of 'shorts' developed in the finished product, as well as making dramatic improvements in the methods used for tempering the glass, which had previously been seen to produce a defect which appeared as a pinhole.

- Improvements have also been made to the overall product to enhance the appearance and market acceptability. These include: (i) increasing the active electrochromic area to enable window manufacturers to install the SageGlass ${ }^{\circledR}$ IGU's into a variety of different framing systems, (ii) implementing a $\mathrm{Pb}$ free solder system for the electrical interconnections, (iii) development of a wire routing scheme to allow installation of SageGlass ${ }^{\circledR}$ units into a variety of different framing systems.

This project has advanced the development of electrochromic glazing significantly, thereby advancing the introduction of the product and all the benefits of such a technology. 